\title{
The origin of ICM enrichment in the outskirts of present-day galaxy clusters from cosmological hydrodynamical simulations
}

\author{
V. Biffi ${ }^{1,2 \star}$, S. Planelles ${ }^{3}$, S. Borgani ${ }^{1,2,4}$, E. Rasia ${ }^{2}$, G. Murante ${ }^{2}$, D. Fabjan ${ }^{5,2}$, \\ M. Gaspari ${ }^{6} \dagger$ \\ ${ }^{1}$ Dipartimento di Fisica dell' Università di Trieste, Sezione di Astronomia, via Tiepolo 11, I-34131 Trieste, Italy \\ ${ }^{2}$ INAF, Osservatorio Astronomico di Trieste, via Tiepolo 11, I-34131, Trieste, Italy \\ ${ }^{3}$ Departamento de Astronomía y Astrofísica, Universidad de Valencia, c/ Dr. Moliner, 50, 46100 - Burjassot (Valencia), Spain \\ ${ }^{4}$ INFN - National Institute for Nuclear Physics, Via Valerio 2, I-34127 Trieste, Italy \\ ${ }^{5}$ Faculty of Mathematics and Physics, University of Ljubljana, Jadranska 19, 1000 Ljubljana, Slovenia \\ ${ }^{6}$ Department of Astrophysical Sciences, Princeton University, Princeton, NJ 08544, USA; Einstein and Spitzer Fellow
}

13 February 2018

\begin{abstract}
The uniformity of the intra-cluster medium (ICM) enrichment level in the outskirts of nearby galaxy clusters suggests that chemical elements were deposited and widely spread into the intergalactic medium before the cluster formation. This observational evidence is supported by numerical findings from cosmological hydrodynamical simulations, as presented in Biffi et al. (2017), including the effect of thermal feedback from active galactic nuclei. Here, we further investigate this picture, by tracing back in time the spatial origin and metallicity evolution of the gas residing at $z=0$ in the outskirts of simulated galaxy clusters. In these regions, we find a large distribution of iron abundances, including a component of highly-enriched gas, already present at $z=2$. At $z>1$, the gas in the present-day outskirts was distributed over tens of virial radii from the the main cluster and had been already enriched within highredshift haloes. At $z=2$, about $40 \%$ of the most Fe-rich gas at $z=0$ was not residing in any halo more massive than $10^{11} h^{-1} \mathrm{M}_{\odot}$ in the region and yet its average iron abundance was already 0.4, w.r.t. the solar value by Anders and Grevesse (1989). This confirms that the in situ enrichment of the ICM in the outskirts of present-day clusters does not play a significant role, and its uniform metal abundance is rather the consequence of the accretion of both low-metallicity and pre-enriched (at $z>2$ ) gas, from the diffuse component and through merging substructures. These findings do not depend on the mass of the cluster nor on its core properties.
\end{abstract}

Key words: galaxies: clusters: general — galaxies: clusters: intracluster medium — methods: numerical

\section{INTRODUCTION}

Galaxy clusters, characterized by deep gravitational potential wells, retain crucial information on the different physical and dynamical processes affecting their stellar, gaseous and dark matter components (see Kravtsov and Borgani 2012, Planelles et al. 2016, for reviews). Therefore, a thorough understanding of the redshift evolution and the distribution of the different cluster components is essential to deepen our comprehension of observed cluster properties. In this sense, X-ray observations of galaxy clusters (see Böhringer and Werner 2010, for a review) have been particularly successful in providing information on the distributions of density, tempera-

* e-mail: biff@@oats.inaf.it

$\dagger$ Einstein and Spitzer Fellow. ture and different metal species within the hot intra-cluster medium (ICM).

The distribution of metals mainly results from the formation of stars in cluster galaxies and from supernova (SN) events. Later on, once metals have been released, thanks to the combined action of a number of processes (such as AGN feedback, galactic winds or ram-pressure stripping, e.g. Churazov et al. 2001; Rebusco et al. 2005. 2006, Simionescu et al. 2008, 2009, Gaspari et al. 2011. 2012, Ettori et al. 2013) they are mixed and redistributed throughout the ICM.

Observations at different redshifts of the ICM spatial distribution and abundance ratios of different metals provide important hints on the number, distribution and metal production efficiency of different SN types. Indeed, whereas light metals (such as, O, $\mathrm{Ne}, \mathrm{Mg}$ or $\mathrm{Si}$ ) are principally released by core-collapse or Type II 
supernovae (SNII), heavier elements (mainly, Fe and Ni) are produced by Type Ia supernovae (SNIa). On the other hand, while both $\mathrm{SNe}$ types contribute similarly to the production of intermediatemass elements (e.g., S, Ar or Ca), SNII and AGB stars contribute to lighter metals, with SNII providing the bulk of $\mathrm{O}, \mathrm{Ne}, \mathrm{Mg}$ and $\mathrm{Si}$ and AGB stars producing the bulk of $\mathrm{C}, \mathrm{N}$ and similar elements (e.g. Böhringer and Werner 2010, Nomoto et al. 2013, de Plaa 2013).

As a further distinction between the two kinds of SN, while SNII (stemming from high-mass short-lived stars) evidence recent star formation events, SNIa (resulting from long-lived low-mass stars) are associated to later metal enrichment. Therefore, the analysis of the ICM metal enrichment and its z-evolution encode crucial information on the cluster star formation history and on the interplay of a number of astrophysical processes shaping observed cluster properties.

In the last years, observations of galaxy clusters with X-ray satellites such as ASCA, BeppoSAX, XMM-Newton or Chandra, have revealed a number of features of the abundance and distribution of different metals in local clusters. Early observations already confirmed that the ICM is characterized by a mean Fe abundance of around $\sim 1 / 3$ the solar value (e.g. Mushotzky et al. 1996 , Finoguenov et al. 2000). Moreover, the iron abundance distribution within galaxy clusters has been shown to depend on the coolcoreness of the considered system: while cool-core (CC) clusters show increasing Fe abundance radial profiles towards their cores, non-cool core (NCC) clusters tend to have a flat distribution out to $\sim 0.4 R_{180}$ (De Grandi and Molendi 2001, De Grandi et al. 2004, Böhringer et al. 2004, Baldi et al. 2007). As a consequence, the levels of core $\left(r<0.1 R_{180}\right)$ entropy and central enrichment are anti-correlated (see, e.g., Leccardi et al. 2010). However, beyond the central core regions, the distribution of metals within the ICM is consistent with a flat and universal radial profile. Besides the abundance of individual elements, the radial distribution of metal abundance ratios, such as $\mathrm{Si} / \mathrm{Fe}$ or $\mathrm{O} / \mathrm{Fe}$, also appears to be nearly flat (see, however, Rasmussen and Ponman 2009, who found a rising $\mathrm{Si} / \mathrm{Fe}$ profile above $\left.\sim 0.1-0.2 R_{500}\right)$, implying a uniform distribution and a similar contribution to metals from SNIa and SNII (e.g. Sato et al. 2008; Sakuma et al. 2011; Matsushita et al.2013).

As for the ICM enrichment history, despite the existing observational difficulties (Molendi et al. 2016), X-ray observations are generally consistent with a non-evolving average metallicity distribution out to $z \sim 1.5$ (e.g. Balestra et al. 2007, Maughan et al. 2008, Baldi et al. 2012, Ettori et al. 2015, McDonald et al. 2016 Mantz et al. 2017).

Most of the results commented so far refer to relatively central cluster regions $\left(r \leqslant 0.2-0.4 R_{180}\right)$. Nevertheless, recent $\mathrm{X}$ ray observations with $S U Z A K U$ of nearby clusters (Werner et al. 2013. Simionescu et al. 2015, Urban et al. 2017, Ezer et al. 2017) and groups (Thölken et al. 2016) have provided precise and wellresolved results on the ICM metallicity distribution out to cluster outskirts, reaching the virial radius and beyond. These observations provide a uniform and universal metal distribution in the outer regions, suggesting an early $(z \geqslant 2)$ enrichment of the ICM (see also Fujita et al. 2008). As a consequence of these observations, we expect a non-evolving cluster metal content at $r \geqslant 0.5 R_{180}$ and out to high redshift. These expectations have been recently confirmed by Mantz et al. (2017), who employed the largest selection of clusters existing so far from X-rays and SZ surveys within $z<1.2$. Contrarily to the innermost cluster volume (e.g. de Plaa et al. 2007 . de Plaa 2013, Mernier et al.2016a b), setting precise constraints on the ICM metallicity in cluster outskirts still represents a challenge since these regions are characterized by a low surface brightness and can be affected by a significant background contamination and multi-temperature gas (e.g. Molendi et al. 2016). Therefore, to explore in more detail the periphery of clusters it will be necessary the use of next-generation X-ray missions, such as Athend ${ }^{1}$ (e.g. Nandra et al.2013), with improved spectral resolutions, larger collecting area, and a precise characterization of the background.

From the theory side, cosmological hydrodynamical simulations represent valuable tools to deepen our knowledge of the ICM chemical enrichment history (see Borgani et al. 2008, for a review). Results from these simulations, including a number of numerical implementations for the AGN feedback, the star formation or the chemical enrichment model, are in line with most of the observational results on the ICM metal content and distribution, its evolution with redshift and the different metal production efficiencies of SNII and SNIa (e.g. Tornatore et al. 2004, 2007; Cora et al. 2008; Rasia et al. 2008; Fabjan et al. 2008, 2010, McCarthy et al. 2010. Planelles et al. 2014, Martizzi et al. 2016, Biffi et al. 2017, Vogelsberger et al. 2017).

In a preliminary analysis, we presented results on the chemical enrichment of the ICM in a set of 29 re-simulated galaxy clusters (Biffi et al.2017), selected from cosmological hydrodynamical simulations performed with an improved version of the SPH code GADGET-3 (Springel 2005). These simulations, accounting for the effects of star formation, chemical enrichment and stellar and AGN feedback, were able to reproduce the coexistence of CC and NCC clusters in a simulated volume (Rasia et al. 2015) and both populations showed a good agreement with observed clusters in terms of entropy and iron abundance profiles, reproducing as well the central entropy-metallicity anti-correlation. In Biffi et al. (2017), we investigated in detail the ICM metal distribution for the same sample of clusters. Specifically, we explored the abundances of different elements, such as $\mathrm{Fe}, \mathrm{Si}$ and $\mathrm{O}$, their spatial and temporal distributions, and the $\mathrm{Si} / \mathrm{Fe}$ and $\mathrm{O} / \mathrm{Fe}$ ratios. We studied the dependencies of these quantities on the different physical processes included in the simulations and on the cluster cool-coreness, discussing as well the different channels for metal production as contributed by SNIa, SNII and AGB stars. In general, our results were consistent with observations of low-redshift massive clusters from the core out to the outskirts.

In the present paper, we complete the analysis presented in Biffi et al. (2017) and we go a step further by investigating in detail the chemical and dynamical evolution of the ICM metal-rich gas populating the outskirts of present-day galaxy clusters. In order to do so, we track back in time the origin of ICM metals found in cluster outskirts at $z=0$. Recalling the importance of iron as a tracer of the ICM metal enrichment level in X-ray observations of clusters, the chemical properties of the tracked gas will be traced by means of its iron abundance. Being our simulations particle-based, it is also straightforward to follow the particles selected at $z=0$ and spatially trace them back, recording the contribution to their metal content due to different enrichment sources (i.e. SNII, SNIa, AGB stars).

The aim of this analysis is to address a number of questions related to the connection between different feedback and enrichment sources, the origin of the gas located in the outskirts of clusters at $z=0$ and contributed by a particular stellar source, or the dynamical evolution of different metal species depending on their main contributing source.

1 http://www.the-athena-x-ray-observatory.eu 
The paper is organized as follows. In Section 2 we briefly describe the main numerical features of our simulations, the chemical evolution model employed and the set of clusters to be analyzed. Section 3 explores the origin and the spatial and temporal evolution of the metal-rich gas located in cluster outskirts at $z=0$. The contribution to the metal enrichment by different sources, such as SNIa, SNII and AGB stars, is also tracked in the simulations and discussed in Section 4 Finally, in Section 5 , we discuss and summarize our main results.

\section{NUMERICAL SIMULATIONS}

The following analysis is performed on a representative subsample of simulated galaxy clusters, analysed also in Biffi et al. (2017). These are part of a set of zoom-in re-simulations of 29 Lagrangian regions extracted from a parent cosmological DM-only simulation, centered on 24 massive clusters $\left(M_{200}>8 \times 10^{14} h^{-1} \mathrm{M}_{\odot}\right)$ and 5 group-size systems $\left(10^{14}<\left[M_{200} / h^{-1} \mathrm{M}_{\odot}\right]<4 \times 10^{14}\right)$, and re-simulated at higher resolution including baryons (see Bonafede et al. 2011). The parent cosmological volume is $1 h^{-3} \mathrm{Gpc}^{3}$ and adopts a $\Lambda$ CDM cosmological model with $\Omega_{m}=0.24, \Omega_{b}=0.04$, $H_{0}=100 \times h=72 \mathrm{~km} \mathrm{~s}^{-1} \mathrm{Mpc}^{-1}, \sigma_{8}=0.8$ and $n_{s}=0.96$ (consistent with WMAP-7 constraints; Komatsu et al. 2011).

Simulations were performed with a version of the TreePM Smoothed-Particle-Hydrodynamics (SPH) code GADGET3 (Springel 2005), in which the hydrodynamical scheme has been modified in order to improve the SPH capabilities of treating discontinuities and following the development of gas-dynamical instabilities. These modifications include higher-order interpolation kernels and derivative operators as well as advanced formulations for artificial viscosity and thermal diffusion (Beck et al. 2016). The mass resolution of these simulations is $m_{\mathrm{DM}}=8.47 \times$ $10^{8} h^{-1} \mathrm{M}_{\odot}$ and $m_{\text {gas }}=1.53 \times 10^{8} h^{-1} \mathrm{M}_{\odot}$, for DM and gas ${ }^{2}$ particles respectively. The Plummer-equivalent gravitational softening length for DM is set to $\epsilon=3.75 h^{-1} \mathrm{kpc}$ in physical units up to $z=2$ and in comoving units at higher redshifts. For gas, star and black-hole particles it is fixed in comoving coordinates, at all redshifts, to $3.75 h^{-1} \mathrm{kpc}, 2 h^{-1} \mathrm{kpc}$ and $2 h^{-1} \mathrm{kpc}$, respectively.

The zoomed-in re-simulations include a large variety of physical processes describing the evolution of the baryonic component. These comprise heating/cooling from Cosmic Microwave Background (CMB) and from a UV/X-ray time-dependent uniform ionizing background (included as in Haardt and Madau 2001), metallicity-dependent radiative cooling (Wiersma et al. 2009), star formation (Springel and Hernquist 2003) and metal enrichment (Tornatore et al. 2004 2007) from various stellar sources. In the CSF version of these runs, it is included only stellar feedback, in the form of thermal SN feedback and galactic (SN-driven) winds with a velocity of $350 \mathrm{~km} \mathrm{~s}^{-1}$, according to the original model by Springel and Hernquist (2003). The more complete set of simulations (AGN) additionally includes a model for gas accretion onto super-massive black holes $(\mathrm{SMBH})$ powering activegalactic-nuclei (AGN) thermal feedback, presented in Steinborn et al. (2015) (mimicking the boosting action of the chaotic cold accretion process; e.g., Gaspari and Sagdowski 2017).

Results on the thermo- and chemo-dynamical properties of

\footnotetext{
2 The gas mass resolution quoted is the intial value. In fact, gas masses are allowed to vary during the simulation, as a consequence of star formation episodes or accretion of metal mass due to chemical feedback from neighbouring star particles.
}

these simulations have been presented in a series of recent papers (Rasia et al. 2015; Villaescusa-Navarro et al. 2016. Truong et al. 2018, Biffi et al. 2016, Planelles et al.|2017, Biffi et al. 2017), to which we refer the interested reader for further details. In particular, we have shown that the AGN runs of the 29 most massive clusters at the center of the re-simulated Lagrangian regions reproduce the observed diversity between $\mathrm{CC}$ and NCC populations, with consistent proportions between the two classes and gas entropy profiles in agreement with observational data (Rasia et al. 2015). The central level of entropy also anti-correlates with the core enrichment level (Biffi et al. 2017), as expected from observational evidences (e.g. Leccardi et al. 2010), and the ICM thermal pressure, temperature and metallicity radial profiles, as well as scaling relations between global observable properties and mass, are in good agreement with a variety of observational datasets (Rasia et al. 2015, Truong et al. 2018, Planelles et al.|2017, Biffi et al.|2017).

We are currently investigating the main properties of the galaxies in our set of simulated clusters, identified by the SubFind algorithm (Dolag et al.2009). Given the complementarity with respect to the current analysis on the origin of the ICM chemical properties, we briefly summarize here some results, while deferring a detailed study, employing as well higher-resolution simulations, to a future dedicated work. The analysis of the galaxy population in our simulations indicates that there is a positive correlation between the total cluster mass at $R_{500}$ and the stellar mass of the central $30 \mathrm{kpc}$ of the cluster, where the brightest cluster galaxy (BCG) resides, in agreement with observational data (e.g., by Kravtsov et al. 2014). In addition, the stellar mass and oxygen abundance of the star-forming gas in resolved galaxies correlate as expected from observations by, e.g., Tremonti et al. (2004), Sánchez et al. (2013) and Hunt et al. (2016). Specifically, galaxies with stellar masses above $\sim 3 \times 10^{10} \mathrm{M}_{\odot}$ show a similar amplitude and trend with respect to the data. Instead, less massive galaxies are in broad agreement with the observed datapoints in terms of amplitude, given the large scatter between the various datasets, but present a steeper relation: galaxies with stellar masses around $\sim[1-3] \times 10^{10} \mathrm{M}_{\odot}$ are consistent with data by Tremonti et al. (2004) while those with stellar masses around $\sim[0.5-1] \times 10^{10} \mathrm{M}_{\odot}$ are in better agreement with Sánchez et al. (2013) and Hunt et al. (2016).

Similar to recent X-ray observations (Mantz et al. 2017), our simulations also present a metallicity-mass anti-correlation within $0.1 R_{500}$ roughly corresponding to the location of the BCG, whereas on global scales (i.e. within $R_{500}$ ) the ICM metallicity is found to vary very little with the system mass, from group to cluster scales (see Truong et al., in prep.).

Chemical enrichment model. The chemical evolution model implemented in our simulations follows the formulation presented in detail in Tornatore et al. (2004 2007).

We only recall here the main features of this model. Heavy elements are produced through three main enrichment channels, namely by SNIa, SNII and AGB stars, depending on their typical life-times and yields. To this purpose, we assume the initial mass function by Chabrier (2003) as a distribution of initial masses for the population of stars, and the mass-dependent life times by Padovani and Matteucci (1993) to account for the different time scales of stars of different masses. The abundance of the various metal species produced during the evolution of a stellar particle is then estimated by considering different sets of stellar yields for SNIa, SNII and AGB (namely Thielemann et al. 2003. for SNIa; Woosley and Weaver 1995 combined with those by Romano et al. 2010 for SNII; Karakas 2010, for AGB stars). Once metals are produced, they are distributed from stars to surrounding gas parti- 


\begin{tabular}{lcccc}
\hline cluster & $\begin{array}{c}M_{\text {vir }} \\
{\left[10^{14} h^{-1} \mathrm{M}_{\odot}\right]}\end{array}$ & $\begin{array}{c}R_{\mathrm{vir}} \\
{\left[h^{-1} \mathrm{Mpc}\right]}\end{array}$ & $\begin{array}{c}R_{200} \\
{\left[h^{-1} \mathrm{Mpc}\right]}\end{array}$ & $\begin{array}{c}\text { cool- } \\
\text { coreness }\end{array}$ \\
\hline D2 & 5.26 & 1.70 & 1.19 & $\mathrm{CC}$ \\
D3 & 6.53 & 1.83 & 1.28 & $\mathrm{NCC}$ \\
D6 & 15.41 & 2.43 & 1.69 & $\mathrm{NCC}$ \\
D10 & 15.46 & 2.43 & 1.65 & $\mathrm{CC}$ \\
\hline
\end{tabular}

Table 1. Main properties of the four systems analysed, at $z=0$. From left to right, the columns report the cluster id, viral mass $\left(M_{\mathrm{vir}}\right)$, virial $\left(R_{\mathrm{vir}}\right)$ and $R_{200}$ radii, and the $\mathrm{CC} / \mathrm{NCC}$ classification based on the core thermal properties.

cles by smoothing them on the SPH kernel, consistently with the other thermodynamical quantities, while no process of metal diffusion is explicitly included in the simulations. Previous studies in the literature have investigated the influence of changing the details of the metal spreading on the final enrichment pattern in simulated clusters (e.g. Tornatore et al. 2007, Wiersma et al. 2009). From various tests presented in Tornatore et al. 2007 for the same chemical evolution model used in the current simulations, we conclude that changes in the numerical details of the metal injection scheme (e.g. number of neighbouring particles or weighting scheme for the metal diffusion to the gas) have only a minor impact on the resulting star formation history, stellar population and chemical enrichment pattern. The differences, in particular, are smaller than the scatter from cluster to cluster. Moreover, for a given scheme of metal spreading, the final enrichment level of the simulated clusters is more strongly influenced by the physical processes included in the smulations, such as the energy feedback (see Fabjan et al.2010. Planelles et al.|2014, Biffi et al.|2017).

We follow the production and evolution of 15 chemical species: $\mathrm{H}, \mathrm{He}, \mathrm{C}, \mathrm{Ca}, \mathrm{O}, \mathrm{N}, \mathrm{Ne}, \mathrm{Mg}, \mathrm{S}, \mathrm{Si}, \mathrm{Fe}, \mathrm{Na}, \mathrm{Al}, \mathrm{Ar}$ and $\mathrm{Ni}$. For each gas particle, we can trace not only the chemical composition, but also the fraction of each metal which is produced by the three enrichment sources (SNIa, SNII, AGB).

In order to investigate the chemical enrichment of the ICM in the simulated galaxy clusters we explicitely compute the mass fractions of all the metals in the gas - also distinguishing among the enrichment sources. We will also study the iron abundances, since iron is typically used as metallicity tracer in cluster X-ray observations. For this, we will provide iron abundances in units of its solar value $\left(4.68 \times 10^{-5}\right.$, iron number fraction relative to hydrogen $)$ according to Anders and Grevesse (1989) (hereafter ANGR89).

Dataset. The following analysis has been performed on the representative subsample of 4 clusters (already presented in Biffi et al. 2017), chosen to include two massive and two smaller systems, in both cases comprising one $\mathrm{CC}$ and one NCC cluster. Results will be shown and discussed in particular for one study-case cluster (D2), that is a small CC object. Similar results were found for all the four systems, essentially with no dependence on either mass or cool-coreness (see discussion in Appendix A). The global properties of the four clusters, namely virial mass and radius, $R_{200}$ radius ${ }^{3}$ and cool-core or non-cool-core (CC/NCC; see

\footnotetext{
3 The cluster virial radius, $R_{\mathrm{vir}}\left(R_{200}\right)$, is defined as the radius enclosing an average overdensity $\Delta_{\text {vir }}(200)$ times the critical cosmic density, $\rho_{c}(z)=3 H(z) /(8 \pi G)$, as predicted by the spherical collapse model (e.g. Bryan and Norman 1998). The virial mass $\left(M_{\mathrm{vir}}\right)$, correspondingly, is the mass enclosed within $R_{\mathrm{vir}}$.
}

Rasia et al. 2015 for details on the classification criteria) classification are listed in Table 1 , for the reference AGN simulation set.

\section{METAL ENRICHMENT OF CLUSTER OUTSKIRTS}

The metal enrichment of the ICM in cluster outskirts is observed to be relatively homogeneous, as shown by the flattening of the metal abundance radial profiles at large cluster-centric distances (e.g. Leccardi and Molendi 2008, Werner et al. 2013, Simionescu et al. 2015, Urban et al. 2017, Mernier et al. 2017). This is also found in hydrodynamical simulations of galaxy clusters, when the feedback from AGN is included (see Biffi et al.2017). In Fig. 1. we show the comparison between the median Fe-abundance profiles for our sample of 29 simulated clusters, namely the most massive ones in each of the re-simulated Lagrangian regions at $z=0$, for the runs with (AGN; thick black solid line) and without (CSF; thick red dashed line) the inclusion of AGN feedback. These iron profiles are mass-weighted (MW) and three-dimensional, and the shaded areas in the figure mark the dispersion around the median profile, using the $16^{\text {th }}$ and $84^{\text {th }}$ percentiles of the distribution. From the comparison, we note that in the outer regions the median Fe profile in the AGN case is flatter and higher (by a factor of $\sim 1.6$ 2.2 in the range $\sim 0.7-1 R_{200}$ ) than in the CSF one (consistently with results presented in Biffi et al. 2017), with minimal scatter across the sample in both sets of simulations. The flatness of the Feabundance profiles in the outer regions, shown in the AGN clusters, is in agreement with observational findings (see also results in Biffi et al. 2017). In fact, the inset in Fig. 1 shows a comparison between the median Fe-abundance profiles of our sample of 29 clusters in the AGN (thick black solid line) and CSF (thick red dashed line) simulations and the metal abundance measurements of a sample of nearby clusters by Urban et al. (2017) (blue asterisks with error bars - here values from their Table 5 have been rescaled with respect to the solar reference value by ANGR89, for consistency with the rest of our paper). The radial range shown is a zoom onto the intermediate and outer regions, i.e. $r>0.25 R_{200}$, where the observational data by Urban et al. (2017) indicate a relatively flat Fe-abundance level $\left(Z_{\mathrm{Fe}}=0.316 \pm 0.012\right.$ solar, w.r.t. the reference value by Asplund et al. (2009), which correspond to $Z_{\mathrm{Fe}} \sim 0.21$ when the solar abundances by ANGR89 are considered, as it is in our case) across the observed clusters. In order to perform a more faithful comparison (see discussion in Biffi et al. 2017), we show here the emission-weighted (EW) projected median $\mathrm{Fe}$-abundance profiles for our simulated set of clusters. The shaded areas around the median profiles mark the $16^{\text {th }}$ and $84^{\text {th }}$ percentiles of the distributions. At large cluster-centric distances the scatter across the sample is larger than in the case of the MW, 3D profiles. This is essentially driven by the presence of substructures, more numerous in a larger volume and more impacting in the CSF runs for the higher emissivity of these clumps. As a net consequence, the two sets of profiles result to be marginally consistent within 1- $\sigma$. Despite this, we still note that the median trends are very different: significantly flatter and higher for the AGN clusters and steeper and lower for the CSE ones. In this respect, the AGN median profile shows a remarkable agreement with the observational data points, in particular for $r>0.5-0.6 R_{200}$.

By means of a representative study case from the AGN sample - D2, whose Fe-abundance profiles are marked with the thin lines in Fig. 1- we investigate in this section the details and origin of the ICM chemical enrichment in the cluster outskirts at $z=0$ and the role of AGN feedback in establishing this pattern. 


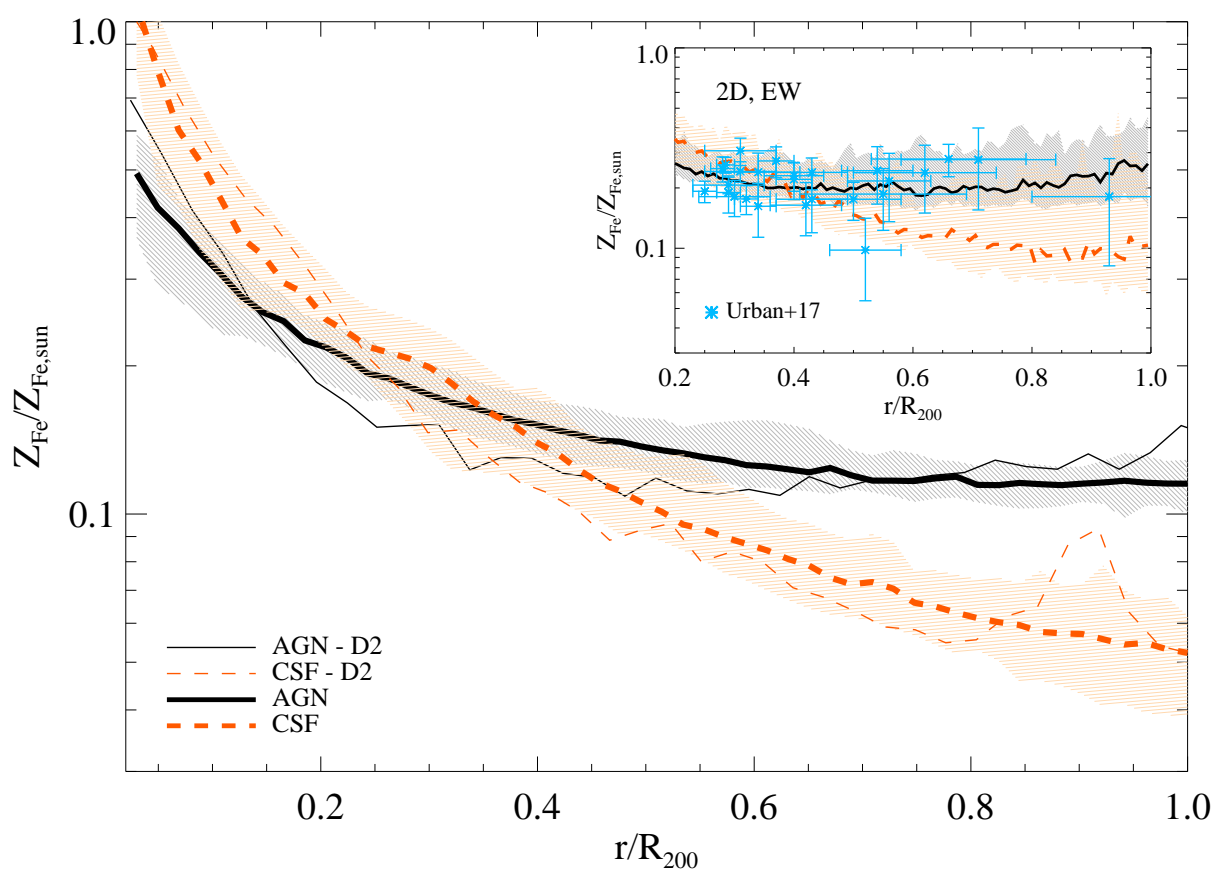

Figure 1. Comparison between the median Fe-abundance profiles (w.r.t. solar values by ANGR89) of the 29 most massive haloes in our set of simulated regions in the two simulation runs with ('AGN') and without ('CSF') the treatment for AGN feedback. Thick lines stand for the median profiles of the whole simulated sample, whereas thin lines represent the corresponding profiles of the region D2. These Fe-abundance profiles are mass-weighted and three-dimensional. The shaded areas around the median profiles represent the $16^{\text {th }}$ and $84^{\text {th }}$ percentiles of the distributions. Top-right inset: Comparison between the emissionweighted, two-dimensional median Fe-abundance profiles of our sample of 29 most massive haloes in the AGN and CSF simulations (see main legend) and the abundance measurements in the outskirts $\left(r>0.25 R_{200}\right)$ of a sample of nearby clusters by Urban et al. (2017) (blue asterisks with error bars).

Specifically, we select the hot-phase gas residing in the external region of the cluster, within the spherical shell comprised between $0.75 R_{200}$ and $R_{200}$, at the present time $z=0$. In order to select the hot-phase ICM at $z=0$, we exclude from our computation those gas particles that have temperature below $3 \times 10^{4} \mathrm{~K}$ or a cold-gas component greater than 10 per cent. Additionally, we exclude explicitly the gas that is in the star-forming phase, i.e. that with density greater than the star-formation density threshold adopted in our code $\rho_{\text {sf_th }}=0.1 \mathrm{~cm}^{-3}$. In the following, this gas - or a subselection of it depending on its $z=0$ iron abundance - will be tracked back in time in order to investigate its chemical properties at higher redshifts, its origin and spatial distribution. For such analysis no restriction on the thermal phase of the tracked gas at redshift $z>0$ will be made (and MW abundances will be always weighted by the total gas mass instead of the hot-phase mass only, for this reason). Averages will be computed considering the MW value, with the purpose of exploring the intrinsic enrichment properties of the tracked gas and not of comparing X-ray-like estimates against observed data. In the following, the main halo at any redshift $z>0$ is the main progenitor of the cluster considered at $z=0$.

With this analysis we intend to explore directly the details of the chemical and dynamical evolution of the ICM in the periphery of present-day clusters. In particular, this will help to answer some key questions like: where does the metal-rich gas in the outskirts of local clusters come from? Is the chemical enrichment of the ICM in these outer regions mostly happening in situ or does it require some pre-enrichment at early epochs? How did different enrichment sources, like SNIa and SNII, contribute to the final enrichment pattern?

\subsection{Origin of the gas in the cluster outskirts at $z=0$}

The hot, diffuse gas selected at $z=0$ in the outer region of the D2 cluster, comprised between $0.75 R_{200}$ and $R_{200}$ is characterised by a large distribution, as it can be seen from Fig. 2, where the median $Z_{\mathrm{Fe}}$ value (blue, dotted line - with the shaded area indicating $\pm 25 \%$ from this value), together with the $16^{\text {th }}$ (red, dashed line) and the $84^{\text {th }}$ (black, dashed line) percentiles are also reported. From this distribution, we notice that the gas in the outskirts of our simulated cluster is skewed towards high iron abundance values, with a low- $Z_{\mathrm{Fe}}$ tail and two main peaks, a broad one around $\sim 10^{-3}-10^{-2} Z_{\mathrm{Fe}, \odot}$ and a second peak around solar values. This

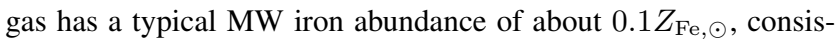
tent with the profiles shown in Fig. 1. We note that the value of the MW Fe abundance, marked with a green solid line, is very close to the $84^{\text {th }}$ percentile of the distribution. This means that most of the mass of the hot gas component is associated to the high-metallicity component of the distribution. A deeper discussion of the distribution shown in Fig. 2 is presented in Section 3.3

In the following, we study the spatial and chemical evolution of this hot gas located in the outskirts of the main halo of D2 at $z=0$. To this purpose, we track the selected gas back in time up to redshift $z=2$, and analyse its spatial distribution and metallicity (primarily using the Fe abundance, $Z_{\mathrm{Fe}}$ ). The map of MW iron abundance $\left(Z_{\mathrm{MW}, \mathrm{Fe}}\right)$, for the tracked gas is shown in Fig. 3 Qualitatively, particles within the cluster outskirts at $z=0$ are located, at higher redshifts, quite far from their current positions, namely at distances corresponding to several times the virial radius of the main cluster progenitor (marked in each map). The observed spatial evolution is the result of a highly dynamical process, where 


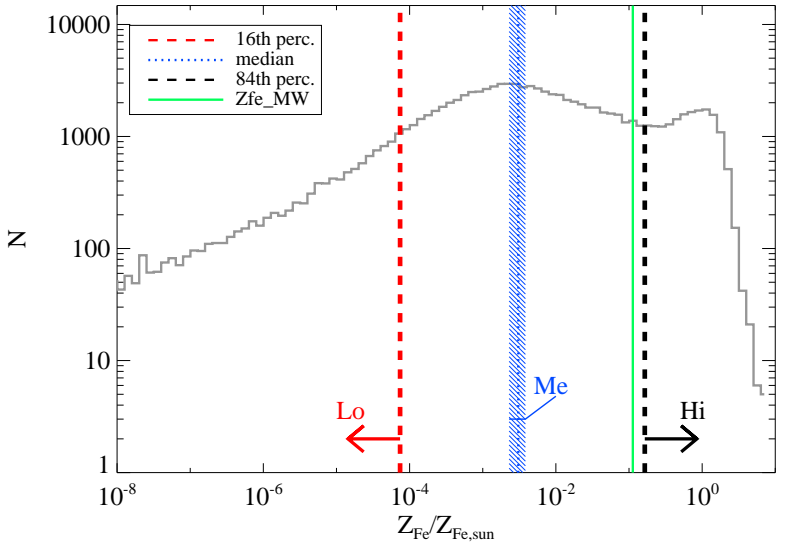

Figure 2. Distribution of the Fe abundance (w.r.t. solar values by ANGR89) for the hot-phase gas particles selected to reside within $[0.75-1] R_{200}$ at $z=0$. As specified in the legend, vertical lines mark: the $16^{\text {th }}$ percentile (red, dashed), the median value (blue, dotted - with the shaded area indicating $\pm 25 \%$ from this value), the MW value (green, solid line) and the $84^{t h}$ percentile (black, dashed) of the $Z_{\mathrm{Fe}}$ distribution. In this figure we also indicate the three gas subselections considered in Section 3.2

the merging of substructures and the gas accretion onto the main central halo determine the final enrichment pattern.

A more quantitative description of this evolution is provided in Fig. 4. where we show the percentage of the tracked gas that resides, at a given redshift $z$, inside the virial radius of the main halo (thick asterisks), within the virial radius of any surrounding halo more massive than $10^{11} h^{-1} \mathrm{M}_{\odot}$ (thin asterisks) or outside of them all (empty circles), either in very small haloes or in the diffuse component. The chosen mass threshold of $10^{11} h^{-1} \mathrm{M}_{\odot}$ allows us to restrict only to haloes that are resolved with a reasonable number of particles. Given our current resolution, we merely consider the haloes and their virial boundary to locate the origin of the tracked gas, while a detailed analysis of these haloes is not allowed, especially at high redshift.

In the lower inset of Fig. 4 we also show the corresponding MW iron abundance, $Z_{\mathrm{MW}}$, Fe. From the analysis of this figure we infer several interesting insights. At $z=0.5$, more than $50 \%$ of all the tracked gas particles is still outside of any halo with $M_{\text {vir }}>10^{11} h^{-1} \mathrm{M}_{\odot}$, included the main one, and is rather enclosed in small galaxies or in the form of diffuse gas. This percentage significantly increases up to $\sim 80 \%$ at redshift $z=2$. Essentially, we see that for this cluster the peripheral gas has been accreted primarily from smaller haloes and from the diffuse component in the redshift range $0.5-2$, when in fact the percentage enclosed within intermediate-mass neighbouring haloes remains roughly constant.

Over this whole redshift range, the MW Fe-abundance of the tracked gas that is found within the virial radius of the main progenitor at each redshift $z$ does not change significantly. This balance is reached through a combination of different phenomena: the continuous accretion of metal-poor gas, the accretion of gas that has been significantly enriched at higher redshift and is either diffuse or in very small sub-haloes that merge onto the main cluster, and some residual enrichment from low-redshift star formation or from long-living stars.

Similarly, the MW Fe abundance of the tracked gas that is enclosed within the surrounding haloes is also relatively constant from $z=2$ down to $z=0.25$, despite a higher normalization with respect to the main cluster. We verified and found that the star formation rate (per unit gas mass) for this gas, locked in the surrounding intermediate-mass haloes, is typically higher than for the gas in the main cluster progenitor suggesting that star formation is still ongoing and continuous metal production is consequently more significant. Despite the fact that we are considering here only the gas selected at $z=0$ in the main cluster periphery and tracked back in time, this is consistent with the expectation that lower-mass systems show a slightly higher content of metals, in mass, with respect to the relative total gas mass (see e.g. Yates et al.|2017). Instead, a mild evolution of the MW Fe-abundance of the gas outside of any halo more massive than $10^{11} h^{-1} \mathrm{M}_{\odot}$ (including the main) is observed, as indeed it increases in the range $\sim 0.02-0.15 Z_{\mathrm{Fe}, \odot}$, from $z=2$ to $z=0.07$. This can be explained by the fact that the large amount of diffuse gas at high redshift must be still pristine or at least very poorly enriched, partly diluting the average $Z_{\mathrm{MW}}$,Fe . As redshift decreases, residual star formation and enrichment from SNIa and AGB contribute to pollute the gas and gas depletion due to the accretion onto the main cluster will combine to produce a net increase of the average iron abundance of the gas that remains in the diffuse component.

Nonetheless, we conclude that also the diffuse gas has a typical abundance that is overall similar to the one already within the cluster, and the other haloes, so that the evolution of the outskirts metallicity below $z<2$ is not expected to be significant. The gas, diffuse and within merging haloes, that is continuously accreted by the forming cluster, has in fact a typical iron abundance which varies only mildly with redshift. This picture is also consistent with recent numerical results from cosmological hydrodynamical simulations performed with the moving-mesh code Arepo, as presented in Vogelsberger et al. (2017).

By inspecting the CSF simulation of the same cluster, instead, we find that the ICM in the present-day outskirts presents a different history. Although accreted as well from the surrounding protocluster region, the majority of the tracked gas has lower metal content and the metal-rich gas is remarkably clumpier, especially at high redshifts. This can be inferred from the comparison between Fig. 6, done for the CSF simulation of D2, and Fig. 3 The MW iron abundance of the tracked gas is significantly lower at all redshifts up to $z=2$, being on average around $0.05 Z_{\mathrm{Fe}, \odot}$ for the gas enclosed within the haloes, main or neighbour ones, and about one order of magnitude lower for the gas outside (see Fig. 8). This is consistent with the idea that the enriched gas is mostly confined to SF regions when no AGN feedback is included, since stellar feedback alone is not enough to displace the gas before it is converted into new generation of stars by the very efficient, un-quenched star formation. This difference at high-redshifts is then propagated into lower-redshift profiles and metal distributions, especially at large distances from halo cores.

\subsection{Spatial evolution of metal-rich gas in the outskirts}

Considering the level of ICM chemical enrichment in cluster outskirts, which is more homogeneous and higher when both stellar and AGN feedback are included in the simulations, it is interesting to investigate the origin of this metal content: where does the gas that is highly-enriched at $z=0$ come from, and what was its metallicity at higher redshift?

Given the typical distribution of iron abundance of the selected gas particles at $z=0$, reported in Fig. 2, we want now to compare the enrichment history of the gas showing extreme enrichment levels at $\mathrm{z}=0$ with the average behavior. To this purpose, we select 3 


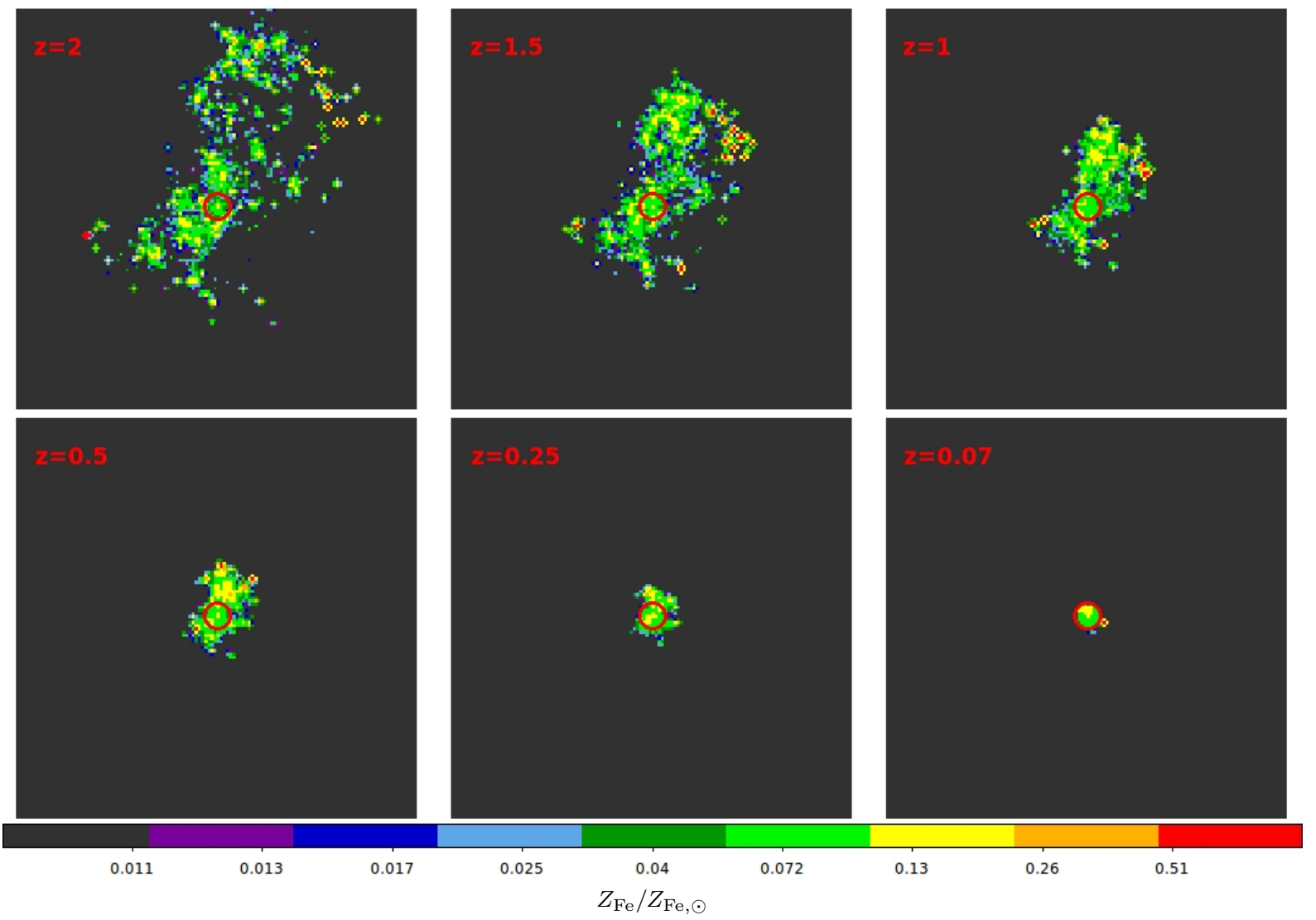

Figure 3. Maps of the spatial distribution, at various redshifts between $z=2$ and $z=0.07$, of the gas residing in the outer regions - i.e. within $[0.75-1] R_{200}$ - of $\mathrm{D} 2$ at $z=0$. The color bar stands for the MW iron abundance, $Z_{\mathrm{MW}}, \mathrm{Fe}$ of the selected particles. The red circle represents the virial radius of the main halo, $R_{\mathrm{vir}}$, at any given redshift and each map is $30 R_{\mathrm{vir}}$ per side.

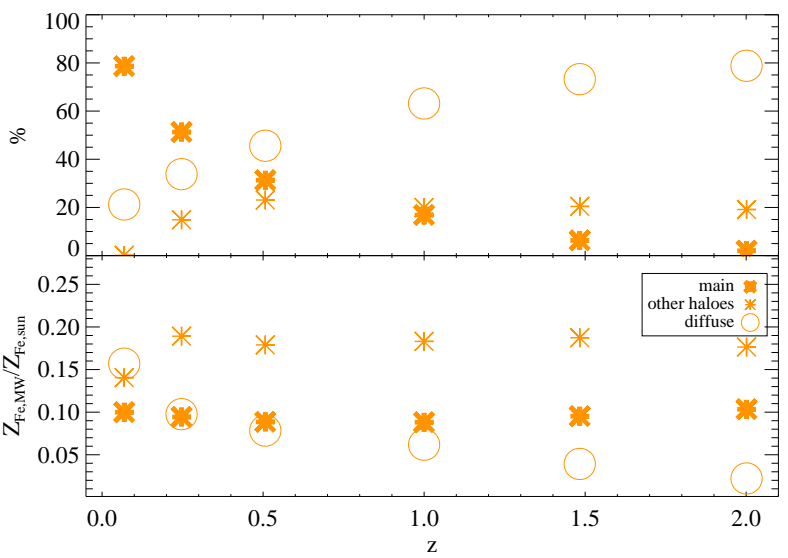

Figure 4. Top: percentage of the tracked particles that reside inside the virial radius of the main-halo progenitor, inside the virial radius of any other halo in the re-simulated region with mass $M_{\mathrm{vir}}>10^{11} h^{-1} \mathrm{M}_{\odot}$, or is rather outside of them - marked by different symbols/sizes, as in the legend - at various redshifts $0.07<z<2$. Bottom: MW Fe abundance (in solar units by ANGR89), $Z_{\mathrm{MW}, \mathrm{Fe}}$, for the same selection used in the upper inset.

subsets of gas particles to trace back in time and space. Namely, we consider:

- $\mathrm{Hi}$ : the highest $Z_{\mathrm{Fe}}$ gas at $z=0$, i.e. with $Z_{\mathrm{Fe}}>Z_{\mathrm{Fe}}^{84 t h}$

- Me: the gas with median $Z_{\mathrm{Fe}}( \pm 25 \%)$ at $z=0$

- Lo: the lowest- $Z_{\mathrm{Fe}}$ gas at $z=0$, i.e. with $Z_{\mathrm{Fe}}<Z_{\mathrm{Fe}}^{16 t h}$ where $Z_{\mathrm{Fe}}^{84 t h}$ and $Z_{\mathrm{Fe}}^{16 \text { th }}$ are the values corresponding to the $84^{\text {th }}$ and $16^{t h}$ percentile of the $Z_{\mathrm{Fe}}$ distribution, respectively (the 3 subselections are also marked in Fig. 2). In a similar way to what is done in Fig. 3 we can specifically track the highly enriched gas selected in the cluster outskirts at $z=0$ ( $\mathrm{Hi}$ gas particles) back in time up to $z=2$. In this way, we explore its spatial distribution with respect to the main progenitor and the surrounding haloes in the region, and its metallicity at higher redshift. The map of the spatial distribution and the MW iron abundance for the $\mathrm{Hi}$ tracked gas is shown in Fig. 5 In general, we note that, even at high redshift, there are high- $Z_{\mathrm{Fe}}$ peaks far beyond the boundary of the main halo, whose virial radius at any corresponding redshift is delimited by the black circle. The global similarity between these maps and those in Fig. 3 indicates that also the high $-Z_{\mathrm{Fe}}$ gas residing in the cluster outskirts at $z=0$ has been accreted from the large-scale structure around the main halo, during the collapse and accretion of neighbouring haloes. Peaks of iron abundance are present already at $z \gtrsim 1$ and located in the map far away from the central progenitor (at distances up to $\sim 10 R_{\text {vir }}$ ).

Similarly to Fig. 4, we can distinguish the accretion history and chemical evolution depending on the final metal content and we show these results for the $\mathrm{Hi}, \mathrm{Me}$, and $\mathrm{L} \circ$ gas components separately in Fig. 9 (from top to bottom, respectively). Also in this case, we explore the spatial origin of the tracked particles and quantify whether at any given redshift up to $z=2$ they were residing already within the main progenitor, within any other surrounding halo more massive than $10^{11} h^{-1} \mathrm{M}_{\odot}$ or rather outside of them all.

From the upper inset in each panel we can infer that the accre- 

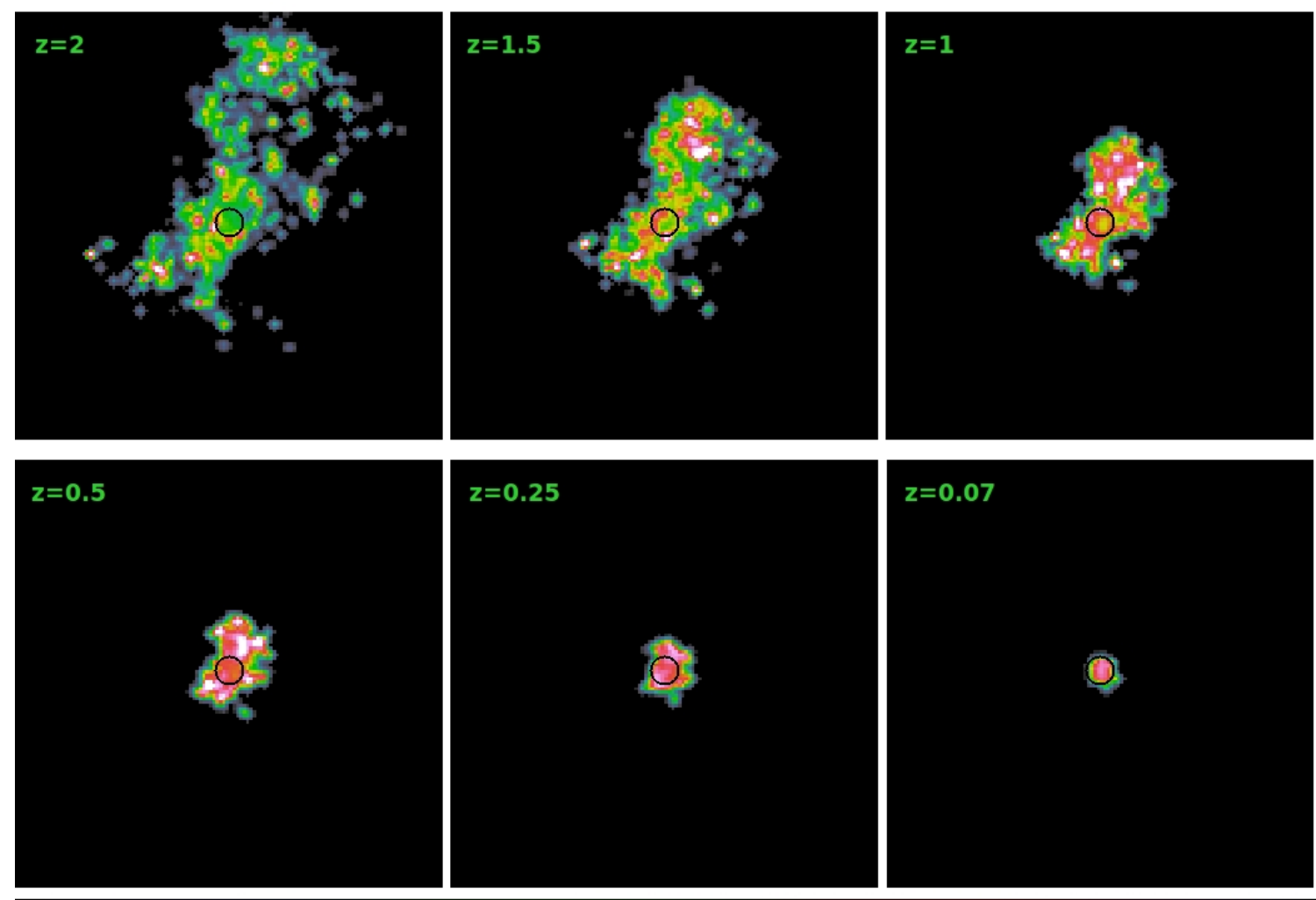

0.11

0.21

0.31

0.41

0.51

0.6

0.8

0.9

$$
Z_{\mathrm{Fe}} / Z_{\mathrm{Fe}, \odot}
$$

Figure 5. Maps of the spatial distribution, at various redshifts between $z=2$ and $z=0.07$, of the high- $Z_{\mathrm{Fe}}$ gas (Hi) residing in the outer regions - i.e. within [0.75-1] $R_{200}-$ of D2 at $z=0$. The color bar stands for the MW iron abundance, $Z_{\mathrm{MW}}$,Fe of the selected particles. The red circle represents the virial radius of the main halo, $R_{\mathrm{vir}}$, at any given redshift and each map is $30 R_{\mathrm{vir}}$ per side.

tion history of these three different components has been different. Namely, the gas that is very poorly enriched at $z=0$ ( Lo subsample, in the bottom panel) has been accreted more recently to the main halo, being for the major part in the diffuse component (more than $\sim 80 \%$ down to redshift $z=0.25$ ). This is the pristine gas that fills the space between the haloes and which has been mostly far from active star formation regions where metal pollution occurs. In fact, its typical $\mathrm{Fe}$ abundance is one order of magnitude smaller when it is still in the diffuse component than when it is already included within one halo, either main or neighbouring (see Fig.9(c) lower inset). In summary, it has a very poor metal content at $z=0$, and has been accreted only recently.

As for the gas with Fe abundance close to the median of the distribution at $z=0$, we note from Fig. 9(b) that its accretion onto the main halo has been smoother over time. A small percentage was residing in the main progenitor already at redshift $z>1$, and a similarly small percentage was also distributed among the surrounding haloes. This increases up to $\sim 20 \%$ by $z \sim 1$, as this gas gets accreted, and then continues to increase for the main progenitor only, as the surrounding haloes themselves merge onto the main cluster at lower redshifts, i.e. $z \lesssim 0.5$. This component, eventually populating the broad peak in the $z=0$ distribution around $\sim 10^{-3}-10^{-2} Z_{\mathrm{Fe}, \odot}$ (see Fig. 2), continues to increase its metallicity from very low values at $z=2\left(\sim\right.$ few $10^{-5} Z_{\mathrm{Fe}, \odot}$ if diffuse, or $\sim$ few $10^{-4} Z_{\mathrm{Fe}, \odot}$ if already included within some halo). This modest level of metal enrichment can mostly be ascribed to the pollution from long-living stars or residual star formation episodes during the cosmic time between $z=2$ and $z=0$, rather than from enrichment within the core of these haloes during the intense peak of star formation activity at $z \sim 2-3$.

A different, interesting picture is outlined by the inspection of the highly-enriched gas component ( $\mathrm{Hi}$ subselection), shown in Fig.9(a) This gas component appears to be accreted onto the main cluster more smoothly in time than the poorly enriched gas, whose majority remains outside the main cluster till very low redshifts. Nevertheless, still $\sim 30 \%$ of this gas is not yet enclosed within any massive halo at $z=0.07$. At higher redshift, the difference in the fraction of gas residing in the outer space and in some halo is not as striking as in the other two (Me and $\mathrm{Hi}$ ) cases. At $z=2$, if enclosed within some halo, it was mostly within intermediatemass surrounding haloes ( $\sim 60 \%$ of it) rather than within the main one $(\sim$ few $\%)$. Even more interestingly, we observe that this gas was already significantly enriched with iron at high redshift, with typical MW Fe abundances of $\sim 0.4-0.5 Z_{\mathrm{Fe}, \odot}$ at $z=2$, if enclosed within some halo. At $z=2$ almost $40 \%$ of it was not enclosed within any halo with mass $M_{\mathrm{vir}}>10^{11} h^{-1} \mathrm{M}_{\odot}$, and yet its MW Fe abundance was already $Z_{\mathrm{MW}, \mathrm{Fe}} \sim 0.35 Z_{\mathrm{Fe}, \odot \text {. This }}$ unbound and enriched gas component was most likely ejected in an earlier phase of AGN activity and then expelled by its galaxy. While the percentage of the gas outside of any of the haloes considered does not diminish significantly, its typical $Z_{\mathrm{MW}}$, Fe increases

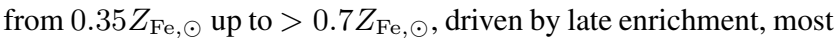
likely due to SNIa rather than fresh star formation generating SNII episodes. 


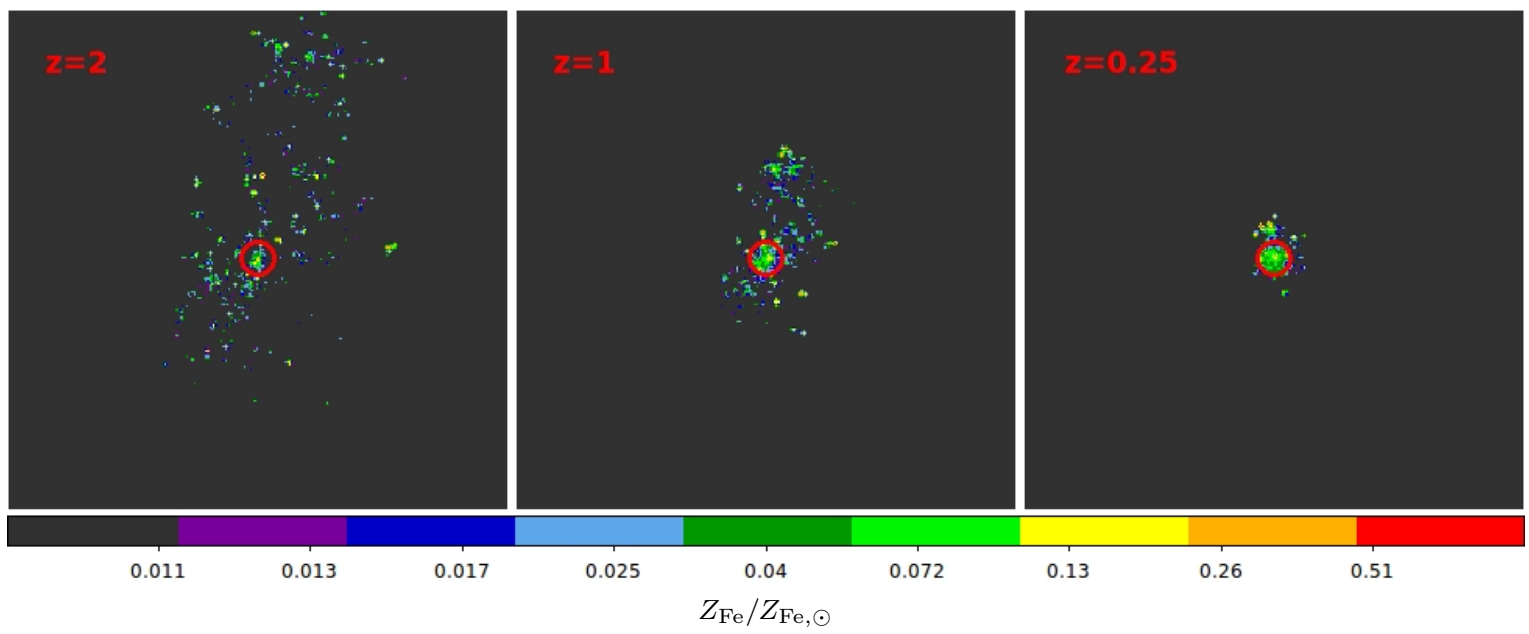

Figure 6. Similar to Fig. 3 but for the tracked gas in the CSF simulation of D2. Here only three redshifts are shown $(z=2,1,0.25)$.

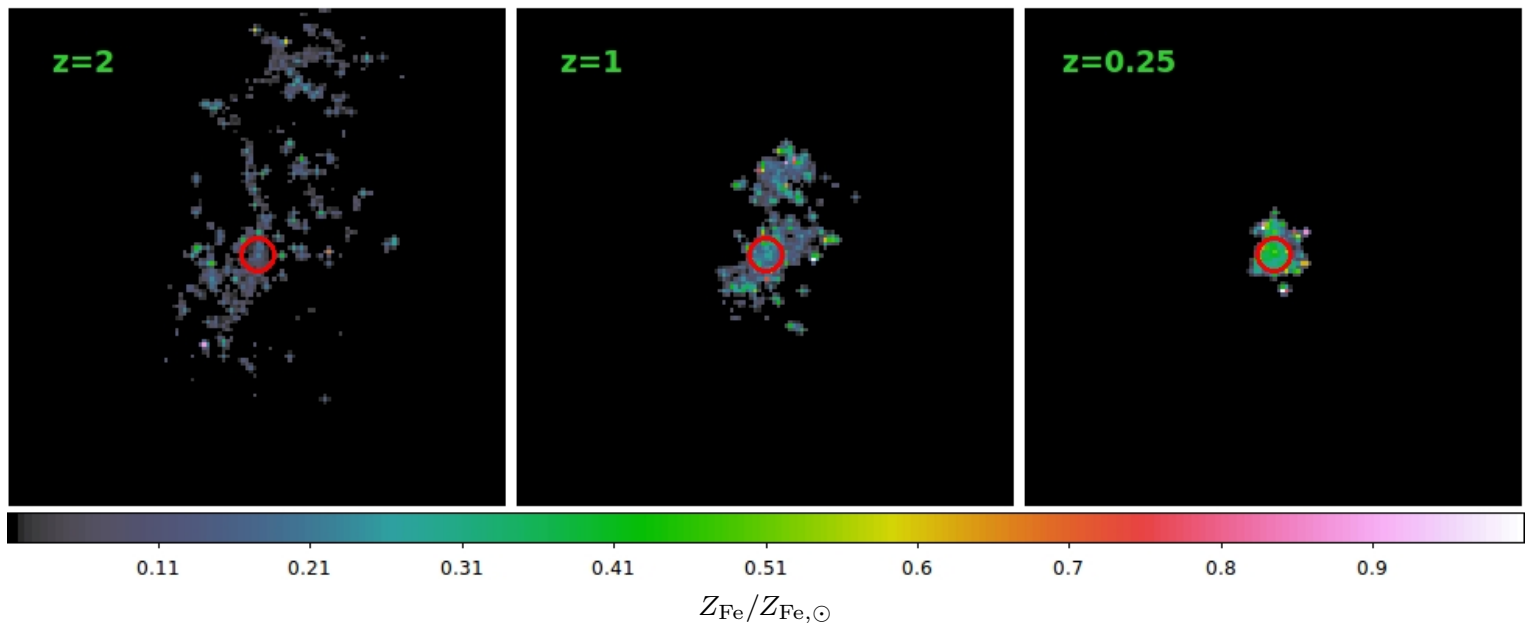

Figure 7. Similar to Fig.5 but for the tracked $\mathrm{Hi}$ gas component in the CSF simulation of D2. Here only three redshifts are shown $(z=2,1,0.25)$.

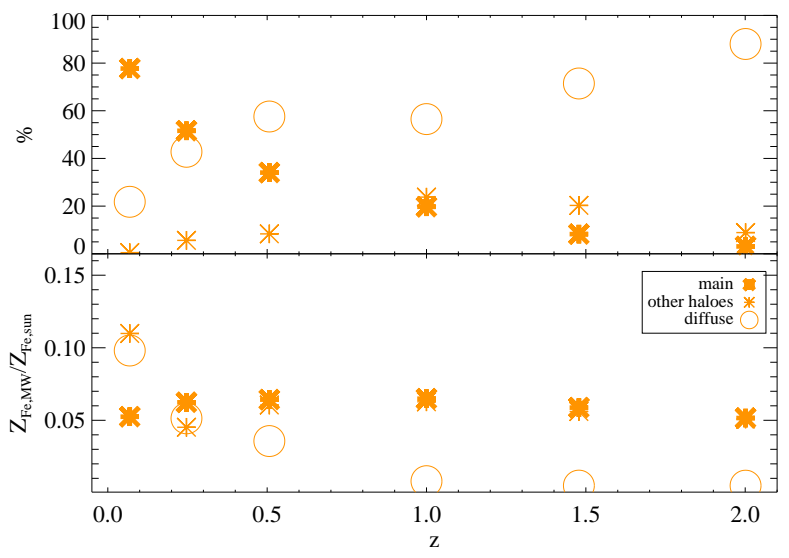

Figure 8. Similar to Fig. 4 but for the CSF simulation of D2.

If we compare the results displayed in Fig. 9 with those in Fig. 4 we infer that the Fe-abundance distribution of the outer gas selected at $z=0$ changes going back in time. On the one hand, we find that the most-enriched particles (Hi) were already Fe-rich at high $z$, both inside and outside the main and surrounding haloes (see Fig.9(a). On the other hand, the majority of all the particles had low $Z_{\mathrm{Fe}}$ and were residing outside the main halo. Indeed, of all the particles tracked, we see from Fig. 4 that only $\sim 20 \%$ of them was already within the main halo or in a surrounding one at $z=2$, and they had in both cases $\mathrm{MW} Z_{\mathrm{Fe}}$ already similar to that at $z=0$. Contrarily, the remaining $\sim 80 \%$ was still to be accreted and had a MW average iron abundance a factor of $\sim 3-4$ lower. Also among the gas in the diffuse component, however, there was already a small fraction of gas that was highly enriched, as visible from the backward tracking of the $\mathrm{Hi}$ subsample, but the $\mathrm{MW}-Z_{\mathrm{Fe}}$ is overall diluted by the low-metallicity gas.

\subsection{Iron abundance evolution of the gas in the $z=0$ outskirts}

The evolution of the $Z_{\mathrm{Fe}}$ distribution for all the gas selected in the cluster outer shell at $z=0$ and tracked back to $z=2$ is shown in Fig. 10 From the figure we note that the high-metallicity peak around solar values is indeed already present at high redshift, albeit lower by a factor of $\sim 2$ in normalization, whereas the strongest difference concerns the low-metallicity tail of the distribution. In particular, the broad peak around $Z_{\mathrm{Fe}} / Z_{\mathrm{Fe}, \odot} \sim 10^{-3}$ vanishes towards higher redshifts, where a greater percentage of the tracked gas has in fact very low iron abundance or is not enriched at all. We verified that the percentage of zero-metallicity gas $\left(Z_{\mathrm{Fe}}=0\right)$ increases going back in time, from $\sim 10 \%$ at $z=0$ to $\sim 80 \%$ at 


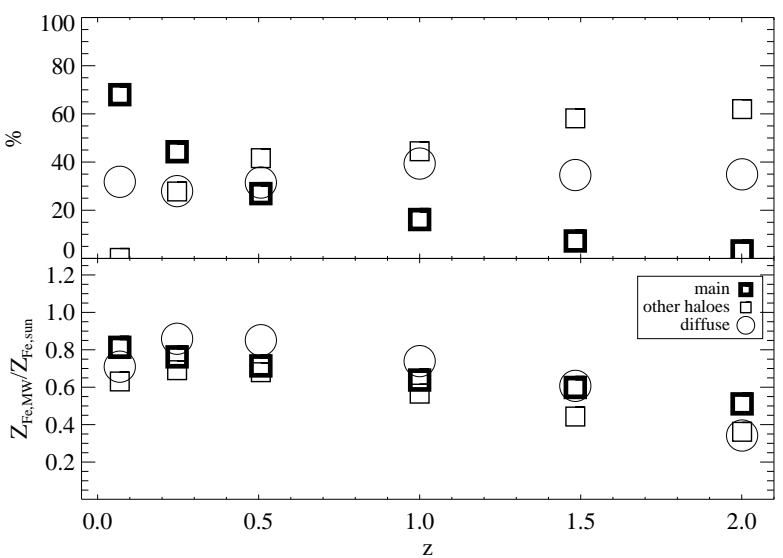

(a) Hi gas.

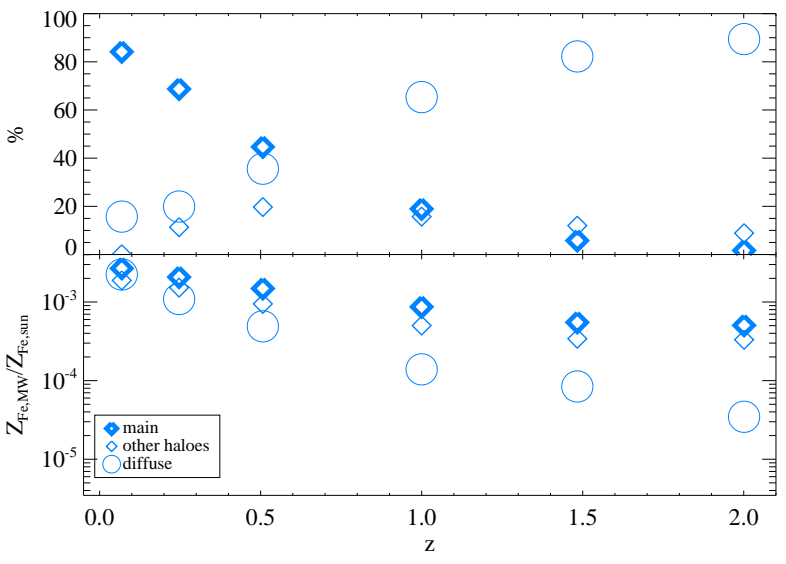

(b) Me gas.

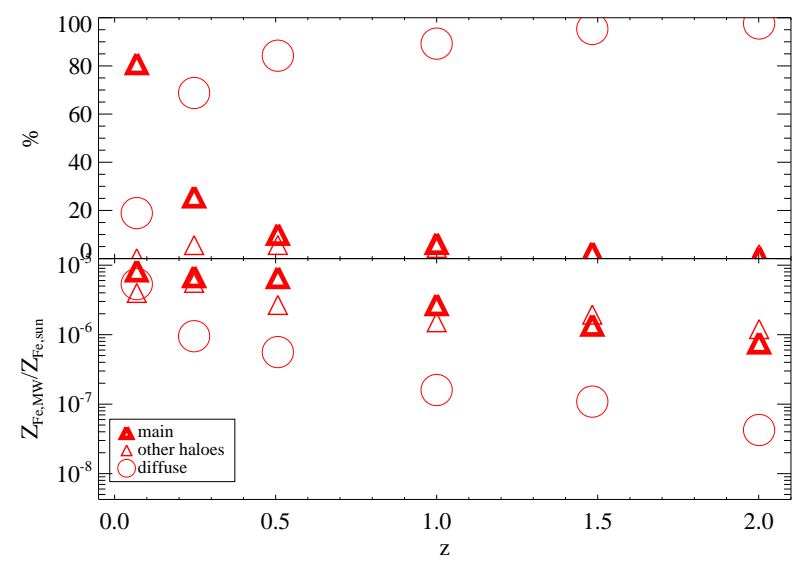

(c) Lo gas.

Figure 9. From top to bottom, same as Fig. 4 for the tracked $\mathrm{Hi}$, Me and Lo gas subselections, respectively.

$z=2$. This broader peak corresponds to the average pollution of gas particles with iron, and essentially results from the combination of the yields and of the distribution of metals from the stars to neighbour gas particles according to the SPH kernel (i.e. depending on their distance from the stellar source).

Concerning the peak around solar values of $Z_{\mathrm{Fe}}$, the presence of a highly-enriched gas component in the cluster outskirts at $z=0$, that has clearly been enriched at $z>2$, is a key imprint

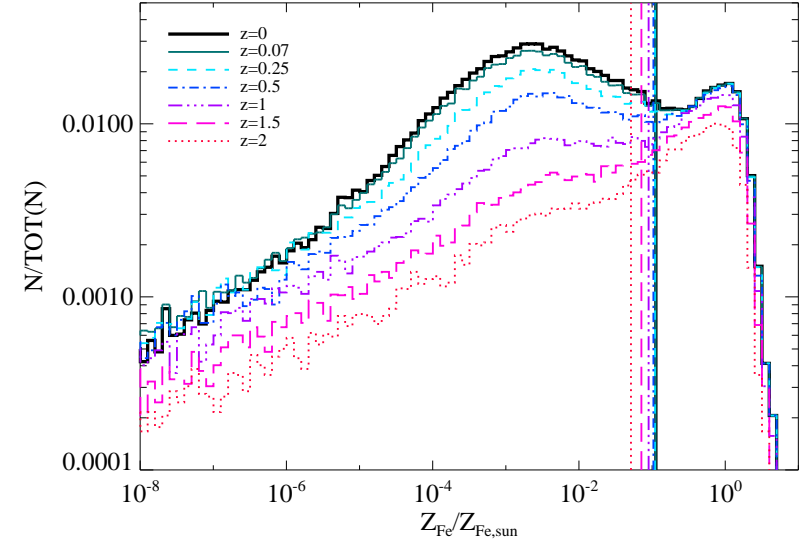

Figure 10. Distribution of the Fe abundance (w.r.t. solar values by ANGR89) for the hot-phase gas particles selected to reside within [0.751] $R_{200}$ at $z=0$ and tracked back in time, at various redshifts up to $z=2$. Vertical lines correspond to the MW value of each distribution.

of early AGN feedback episodes. We remind the reader that in our models we do not include a kinetic feedback so this phenomenon is entirely due to the buoyancy of gas with freshly boosted energy, that is able to displace metal-rich gas out to large distances from the star formation regions, since high-redshift small-mass haloes have shallower potential wells than present-day massive clusters (see also Biffi et al.2017). In fact, a similar analysis on the CSF simulation of the same cluster showed a different distribution for the gas selected in the same outer shell at $z=0$, displayed in Fig. 11 Namely, no secondary peak of the iron abundance distribution is present around solar values of $Z_{\mathrm{Fe}}$, neither at $z=0$ nor at higher redshifts. This indicates that the most-enriched gas is typically confined in the very vicinity of star formation regions and is preferentially converted into newly formed stars, where the majority of the metal content is therefore locked. The lack of a powerful feedback mechanism at high redshift essentially prevents the gas to be moved away before it is again processed into stars. In fact, we verified that a similar tracking of the most enriched gas at $z=0$, in the CSF case, shows lower iron abundances, by a factor of $2-4$ if enclosed within some halo, throughout the redshift range $z=0-2$. If we track the $\mathrm{Hi}$ gas outside any halo more massive than $10^{11} h^{-1} \mathrm{M}_{\odot}$, then the typical $Z_{\mathrm{MW}, \mathrm{Fe}}$ in the CSF run is up to one order of magnitude lower at high redshift. In fact, as previously noted for Fig. 6 this behaviour can also be appreciated from the maps of MW iron abundance in Fig.7 for the $z=0 \mathrm{Hi}$ gas component. The same spatial scale and color code used for the AGN case emphasize how the most iron-rich gas distribution is clumpier and characterised by a lower values of $Z_{\mathrm{MW}, \mathrm{Fe}}$. From both Fig. 6 and Fig. 7 we see that also in the CSF case the metal distribution traces the large scale structure around the main cluster progenitor and the accretion of the neighbouring haloes onto it, but the level of iron abundance is typically lower, with the peaks of high abundance particularly localized. This difference is remarkable especially at high redshift, where on the contrary the iron-rich gas in the AGN simulation, as well as the tracked $\mathrm{Hi}$ component, was already widely distributed across the whole region connecting the main cluster and the surrounding haloes. 


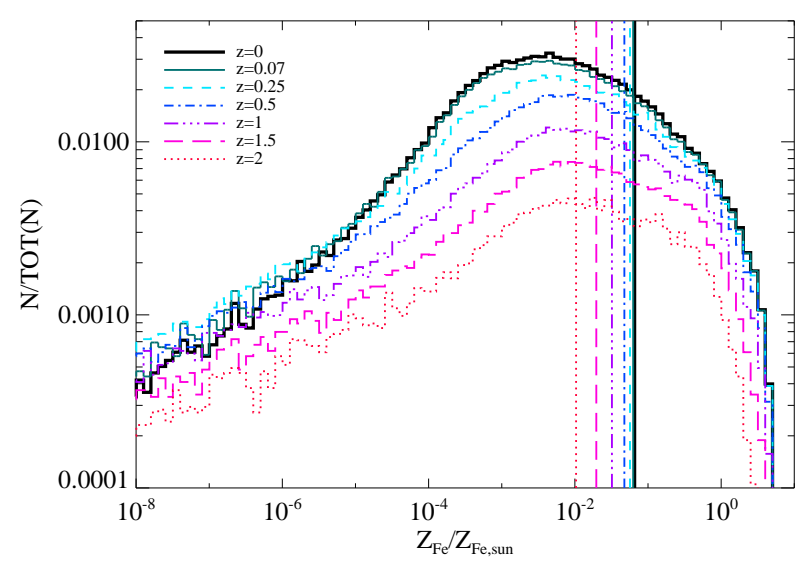

Figure 11. Similar to Fig. 10 but for the CSF simulation of D2.

\section{TRACING THE CONTRIBUTION FROM DIFFERENT ENRICHMENT SOURCES}

In this section we will discuss the origin of the metal content of the gas residing in the cluster outer shell at $z=0$ in terms of the contribution to the enrichment from SNIa and SNII.

In fact, SNII release mainly light metals (such as, $\mathrm{O}, \mathrm{Ne}, \mathrm{Mg}$ or $\mathrm{Si}$ ), whereas heavier elements (like $\mathrm{Fe}$ and $\mathrm{Ni}$ ) are predominantly produced by Type Ia supernovae. Iron is in reality produced by both SNII and SNIa, but with different time-scales and yields, being the contribution of SNIa the most significant especially at later times. Lighter elements such as $\mathrm{C}$ or $\mathrm{N}$ are produced mainly by low- and intermediate-mass AGB stars.

Throughout the cosmic history, different stellar sources contributed to producing metals and polluting the surrounding gas. The specific contribution from SNIa and SNII - and consequently of the AGB stars - can be evaluated directly in our simulation. In Fig. 12 we show the metal mass fraction due to SNIa (solid lines) and SNII (dotted lines) for all the metal-rich gas tracked at various redshifts between $z=0$ and $z=2$. As expected, the contribution from SNII is dominating the metal mass fraction at all redshifts. This means that the majority of metals, in terms of total mass, is produced by SNII, which is in fact consistent with oxygen being the most abundant element in the Universe. On the other hand, the mass of metals produced by SNIa increases with decreasing redshift. In fact, the evolution with redshift of these contributions is opposite and in agreement with the typical life time scales associated to the two stellar sources: overall, the SNIa-fraction increases from $\sim 20$ per cent at $z=2$ to $\sim 30$ per cent at $z=0.07$; differently, the SNII-fraction decreases from $\sim 65$ per cent to $\sim 55$ per cent in the same temporal range. From Fig. 12 one can easily infer that the remaining metal mass is due to AGB stars, whose contribution roughly oscillates around $\sim 15$ per cent. The different symbols used (as in Fig. 4 refer to the location of the tracked gas at the given redshift $z$, so that we can differentiate among that already accreted onto the main cluster, the fraction still enclosed within some other intermediate-mass neighbouring halo and the fraction that is still outside of them all. Interestingly, we find that the trend does not depend significantly on the environment in which the gas is located, suggesting that this result is mainly driven by the time-delay and yields of the enrichment channels followed by our simulations. Indeed, variations among the three sets of values are on average within $10 \%$.

In Fig. 13 we show the contribution from these two main en-

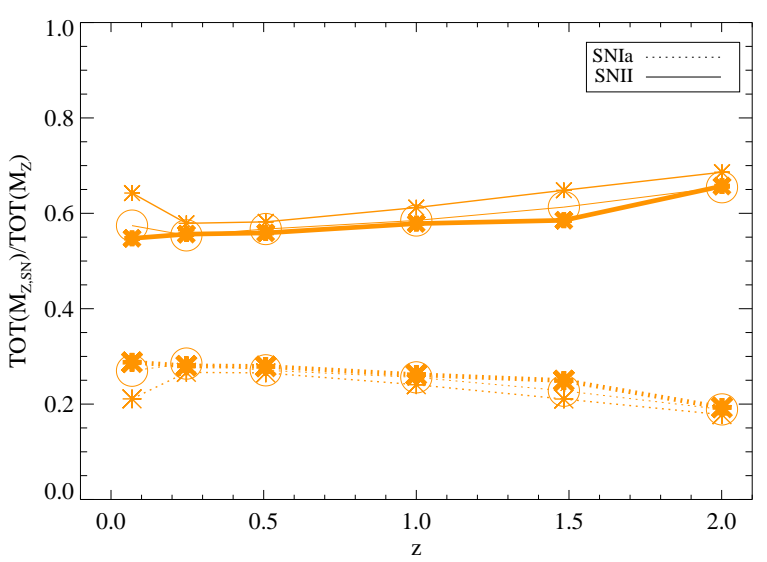

Figure 12. Fraction of the mass of metals produced by SNIa (dotted lines) or SNII (solid lines) with respect to the total mass of metals, for the gas selected at $z=0$ in the spherical shell $[0.75-1] R_{200}$ and tracked back in time up to $z=2$. Different symbols refer to the location of the tracked gas at redshift $z$, namely within the main progenitor (thick asterisks), within the virial radius of any other surrounding halo with $M_{\text {vir }}>10^{11} h^{-1} \mathrm{M}_{\odot}$ (thin asterisks), or outside of them all (empty circles).

richment channels for the subselections of the gas based on its $z=0$ iron abundance ( $\mathrm{Hi}, \mathrm{LO}$ and Me components, from top to bottom, respectively). The $\mathrm{Hi}$ gas component shows very similar results to those discussed for the whole gas in Fig. 12 This suggests that the high- $Z_{\mathrm{Fe}}$ gas - which has been found to have relatively high $Z_{\mathrm{Fe}}$ also at higher redshifts - essentially dominates the mass budget, namely contains the majority of metals in mass. This gas must have been therefore significantly enriched by both SNIa and SNII, since very early times.

Differently, the gas with median or very low $\mathrm{Fe}$ abundance at $z=0$, which has typically even lower abundances at higher redshifts, is characterised by a metal mass content, albeit modest, which is essentially dominated by the contribution from SNIa. In both cases, especially at late times, the SNIa-channel is contributing up to $80 \%$ of the total metal mass because of the longer timescales of their enrichment. The contribution from SNII is basically negligible, except for a $20 \%$ contribution to the enrichment of the Me gas at $z \gtrsim 1$, probably within haloes smaller than those considered here (i.e. with $M_{\text {vir }}<10^{11} h^{-1} \mathrm{M}_{\odot}$ ). Instead, the remaining fraction of metal mass (comprised between $20 \%$ at late times and $\sim 40 \%$ at early times) is due to AGB sources.

SNIa, and in a minor proportion AGB stars, essentially contribute to build up the broad peak centered around $10^{-3} Z_{\mathrm{Fe}, \odot \text {, in }}$ the distribution shown in Fig. 10 This all indicates that the poor enrichment of this gas happened far from SF regions, where the pollution due to short-living SNII is not significant. To a closer inspection, we found in fact that the distribution of the metal-rich particles that we track shows a significant component of quasi-pristine gas that contains only a little amount of metals almost entirely coming from SNIa sources. This gas has very low $Z_{\mathrm{Fe}}$ and has been probably enriched by late SNIa episodes far away from SF regions, given that long-living stars have more time to move away before dying as SNIa and polluting the surrounding medium.

Both Fig. 12 and Fig. 13 remark that the properties of the tracked gas with respect to the enrichment sources are essentially the same independently of whether it is residing within a halo (either the main or a neighbour one) or not, from $z=2$ down to $z \sim 0$. The results obtained with our current modelling of chemi- 


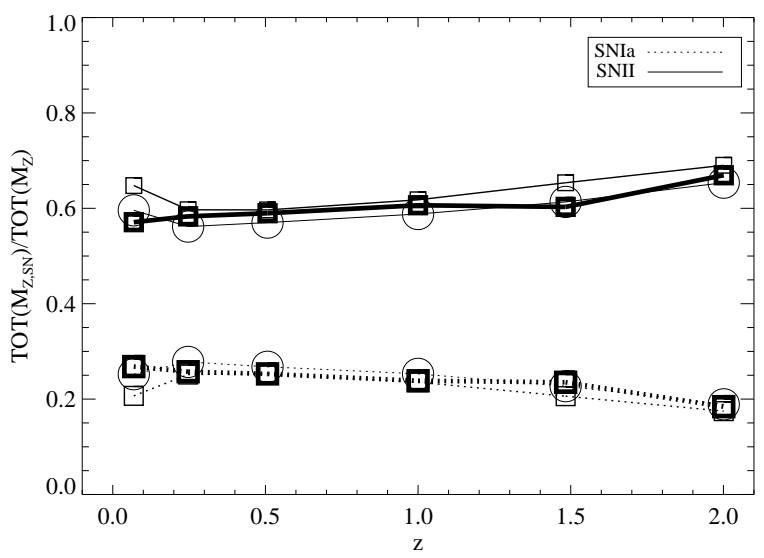

(a) $\mathrm{Hi}$ gas.

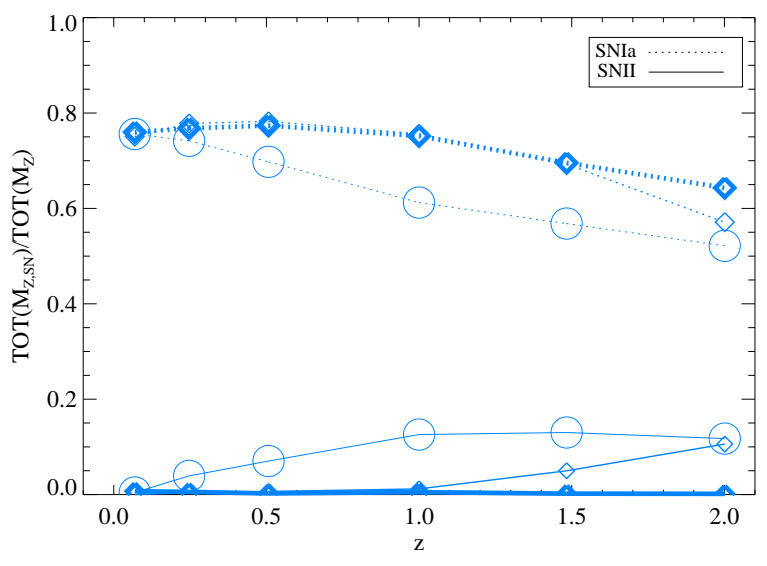

(b) Me gas.

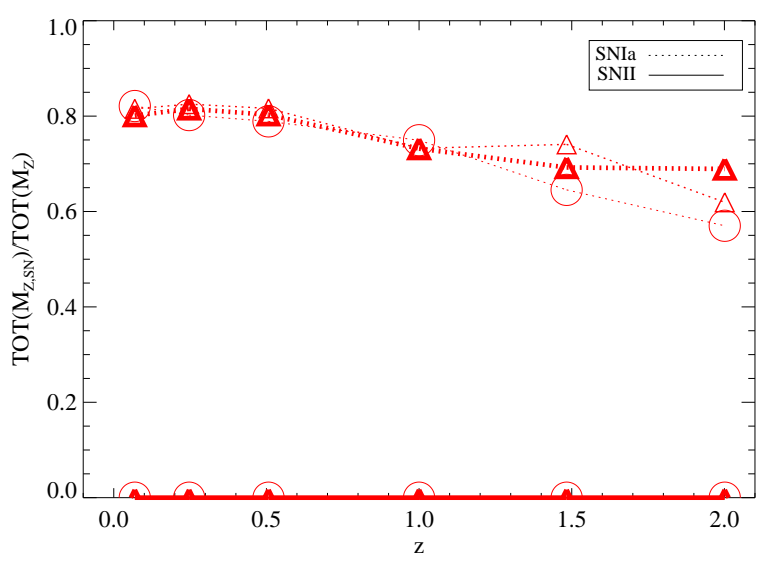

(c) Lo gas.

Figure 13. From top to bottom, same as Fig. 12 for the tracked $\mathrm{Hi}$, Me and Lo gas subselections, respectively. (Symbols refer to different environments as in Figs. 49 and 12 .

cal evolution and AGN thermal feedback, further support the idea that the bulk of the enrichment is not happening "in situ" within the formed clusters, but is rather the result of a more complex and continuous process of accretion of both pristine gas and highly metal-rich gas, that was previously enriched within neighbouring substructures that eventually merge onto the main halo.

\section{SUMMARY AND CONCLUSION}

In this paper we focused on the origin of ICM chemical enrichment in the present-day outskirts of simulated clusters from a set of cosmological, hydrodynamical zoomed-in simulations of galaxy clusters performed with an improved version of the GADGET- 3 code. The simulations include the treatment of a variety of physical processes describing the physics of the baryonic component, among which thermal feedback from AGNs. Results on the thermo- and chemo-dynamical properties of clusters in these simulations have been shown to agree with a variety of observational evidences in a series of recent papers (Rasia et al. 2015. Villaescusa-Navarro et al. 2016, Truong et al. 2018, Biffi et al. 2016, Planelles et al. 2017, Biffi et al. 2017). Here, we traced back in time the hot gas particles that reside in the outskirts of the cluster at $z=0$, defined as the region between $0.75 R_{200}$ and $R_{200}$, and investigated the evolution of their spatial distribution and enrichment level and source, up to $z=2$. We remark that the properties that we discuss are always referred to the gas residing in the present-day outskirts and tracked back in time, only.

With the present analysis we aim at the detailed origin of the remarkably uniform metal enrichment typical of present-day cluster outskirts, which is found both in observations (Werner et al. 2013. Simionescu et al. 2015, Urban et al. 2017) and in simulations (Biffi et al.2017, Vogelsberger et al.|2017). From simulations, we find that the flat metallicity and abundance profiles at large cluster-centric distances turn into steeper and lower-normalization gradients when exclusively stellar feedback is included in the simulations. This is a footprint of the crucial role played by high-redshift AGN feedback in distributing the metal-rich gas far away from star formation regions, out and much beyond the virial radius of surrounding haloes in the proto-cluster environment.

While we presented results for one single study-case cluster, we find that very similar trends are confirmed when different clusters, in terms of mass and cool-coreness, are investigated. This is discussed in Appendix A In particular, we find that the key feature of the present-day iron abundance distribution of the hot gas in the outer cluster shell, namely the secondary peak around solar values, is always present in the four clusters analysed, and already from redshift $z=2$.

We summarize hereafter our main findings.

- A component of the gas selected in the present-day outer shell of the (AGN) cluster is highly enriched with iron, with $Z_{\mathrm{Fe}}$ peaked around solar values. This component dominates the mass-weighted average Fe abundance. We find that this component is also already present at $z=2$ and already with significant enrichment level, while the fraction of median- $Z_{\mathrm{Fe}}$ gas increases, due to the later enrichment.

- Most of the gas in the present-day outskirts has been accreted onto the main cluster only recently. At $z=0.5$, about $\sim 70 \%$ of the gas found in these regions was not yet accreted onto the main halo. At redshift $z \geqslant 1$ more than $60 \%$ of this gas is in the diffuse component or in much smaller haloes.

- The mass-weighted iron abundance typical of this tracked gas is comprised between 0.1 and 0.2 solar, and does not evolve much as long as it is residing in any halo, either main or not, at higher redshifts. The gas in the diffuse component increases its $\mathrm{MW} Z_{\mathrm{Fe}}$

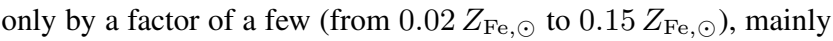
due to the fact that the diffuse component at high redshifts is diluted by a significant fraction of pristine gas.

- From the backward tracking of the gas particles, our results indicate that the highly enriched gas residing in the outskirts at $z=0$ 
was already significantly enriched at higher redshifts up to $z=2$ (when, $Z_{\mathrm{Fe}} \sim 0.4-0.5 Z_{\mathrm{Fe}, \odot}$ ). This gas has been likely enriched at even higher redshifts, during or before the peak of star formation, and was later accreted onto the main cluster, together with more pristine gas, both from the diffuse component and through the merging with surrounding haloes during the cluster mass assembly.

- The chemical enrichment of the tracked gas is overall dominated, in mass, by the metal pollution due to SNII ( 55-65\%, from redshift $z=0$ to $z=2$ ). This is essentially driven by the enrichment characteristics of the most metal-rich gas (for which the SNII metal mass fraction is $\sim 60-70 \%$, in the range $0<z<2$ ). There is however a gas component, the one with median or very low iron abundance, which is mainly enriched by SNIa $(\sim 60$ $80 \%$, from redshift $z=0$ to $z=2)$ - and partially AGB $(\sim 20 \%$ at $z=0$ to $30-40 \%$ at $z=2$ ) - products, indicating that the enrichment, albeit modest, has happened at later times and mostly far from SF regions.

- From the study of the enrichment sources, we note that this shows no significant dependency on the environment where the tracked gas resides at a given redshift. Relative proportions of metal mass fractions for SNIa and SNII are the same for both the gas within some halo (either main or neighbour) or in the diffuse component.

- When we consider the simulation of the same cluster that does not include AGN feedback but only stellar feedback (CSF), we observe a sparser distribution of the Fe-rich gas, when the selected particles at $z=0$ in the outskirts are traced back in time. Also, their typical metal enrichment level is lower and, in fact, their distribution of iron abundances shows no component peaked at solar or higher values, either at $z=0$ where they are selected or at $0<z \leqslant 2$.

The analysis presented in this paper confirms that the relatively high and uniform metallicity of the ICM in the outskirts of nearby clusters, traced by its iron abundance, is the result of the accretion with time of both pristine and already highly enriched gas, from both the diffuse component and from surrounding haloes in the proto-cluster environment that later merge onto the progenitor of the main cluster during its formation and mass assembly. After the high-redshift pollution of the gas with metals, by both SNII and SNIa, mixing and dynamical processes have played a major role in distributing them and in shaping the present-day profiles at large cluster-centric distances. Interestingly, we observe in simulations that at $z=2$ part of the highly enriched gas is diffuse and resides outside of any intermediate-mass halo in the protocluster region. This gas has been expelled out to large distances from the pollution sites at higher redshifts by powerful AGN episodes in small-mass haloes. This can be inferred by the comparison to simulations including only stellar feedback, where in fact the level of ICM enrichment is lower and much more localized, confined to the SF sites (see also Biffi et al.2017).

While the present-day imprints of this pre-enrichment mechanism has already found confirmation in observational evidences, the ideal next step would be to push X-ray observations of the ICM enrichment to the region close or immediately beyond the virial radius or to the diffuse medium between pre-merging systems, and to clusters at higher redshifts. In fact, this will help constraining the widespread distribution of metal-enriched gas in the proto-cluster environment (see also studies on galaxies in dense proto-cluster regions at high redshifts, e.g. Overzier 2016), and the later accretion of these metals to the cluster, rather than the in-situ production at lower redshifts. To this goal, next generation of X-ray satellites with large effective areas and high-resolution spectroscopy, such as Athena 4 (Nandra et al. 2013, Barret et al. 2016), will enable far more detailed observations of the cluster outskirts and reliable measurements of the ICM metallicities.

From the point of view of numerical simulations, we remark here that a number of physical processes - such as dust production and disruption or metal diffusion - are not yet included in this set of simulations and will need to be accounted for in order to pursue further detailed studies on the chemical evolution of galaxy clusters. An additional limitation of the current simulation set is the resolution, which prevents us from resolving the details of the galaxy component in the clusters, especially at high redshift. Although the ICM pre-enrichment picture presented here is consistent with results from independent studies on higher-resolution simulation sets (see recent results by Vogelsberger et al.2017), as a future improvement, we plan to perform and investigate higher-resolution simulations in order to consistently explore the details of the galaxy population within clusters, which interact with the ambient ICM since high redshift and contribute to both energy and chemical feedback (see e.g. Renzini and Andreon 2014). This will allow us to study the details of the star formation history and chemical patterns of the stellar component within galaxies, adding information to the picture discussed in the present analysis.

Combining high resolution and improvements on the physical description of the simulations will be crucial especially for detailed comparisons with observational findings, also of the high-redshift Universe, expected from upcoming missions like Athena.

\section{ACKNOWLEDGEMENTS}

The authors would like to thank V. Springel for allowing us to access the developer version of the GADGET code and L. Steinborn for providing access to the improved AGN feedback model. The authors are thankful to G.L. Granato, C. Ragone-Figueroa, L. Tornatore and K. Dolag for useful discussions during the early stages of this work, and to the anonymous referee for constructive and insightful comments on the manuscript. Simulations have been carried out using Flux HCP Cluster at the University of Michigan, Galileo at CINECA (Italy), with CPU time assigned through ISCRA proposals and an agreement with the University of Trieste. We also acknowledge PRACE for awarding us access to resource ARIS based in Greece at GRNET, through the DECI-13 PRACE proposal. The post-processing has been performed using the PICO HPC cluster at CINECA through our expression of interest. We acknowledge financial support from the PRIN 2015W7KAWC founded by the Italian Ministery for University and Research, the INFN INDARK grant, and "Consorzio per la Fisica" of Trieste. SP acknowledges support from the "Juan de la Cierva" program (ref. IJCI-2015-26656) funded by the Spanish Ministerio de Economía y Competitividad (MINECO) as well as from the MINECO through the grants AYA2013-48226-C3-2-P and AYA2016-77237-C3-3-P and the Generalitat Valenciana (grant GVACOMP2015-227). DF acknowledges financial support from the Slovenian Research Agency (research core funding No. P10188). M.G. is supported by NASA through Einstein Postdoctoral Fellowship Award Number PF5-160137 issued by the Chandra Xray Observatory Center, which is operated by the SAO for and on

\footnotetext{
4 http://www.the-athena-x-ray-observatory.eu/
} 
behalf of NASA under contract NAS8-03060. Support for this work was also provided by Chandra grant GO7-18121X.

\section{REFERENCES}

Anders E., Grevesse N., 1989, Geochim. Cosmochim. Acta, 53, 197

Asplund M., Grevesse N., Sauval A.J., Scott P., 2009, ARA\&A, 47, 481

Baldi A., Ettori S., Mazzotta P., Tozzi P., Borgani S., 2007, ApJ, 666,835

Baldi A., Ettori S., Molendi S., Balestra I., Gastaldello F., Tozzi P., 2012, A\&A, 537, A142

Balestra I., Tozzi P., Ettori S., Rosati P., Borgani S., Mainieri V., Norman C., Viola M., 2007, A\&A, 462, 429

Barnes D.J., et al., 2017, ArXiv e-prints

Barret D., et al., 2016, in Space Telescopes and Instrumentation 2016: Ultraviolet to Gamma Ray, vol. 9905 of Proceedings SPIE, p. 99052F

Beck A.M., et al., 2016, MNRAS, 455, 2110

Biffi V., et al., 2016, ApJ, 827, 112

Biffi V., et al., 2017, MNRAS, 468, 531

Böhringer H., Werner N., 2010, A\&A Rev., 18, 127

Böhringer H., Matsushita K., Churazov E., Finoguenov A., Ikebe Y., 2004, A\&A, 416, L21

Bonafede A., Dolag K., Stasyszyn F., Murante G., Borgani S., 2011, MNRAS, 418, 2234

Borgani S., Fabjan D., Tornatore L., Schindler S., Dolag K., Diaferio A., 2008, SSR, 134, 379

Bryan G.L., Norman M.L., 1998, ApJ, 495, 80

Chabrier G., 2003, Publ. Astr. Soc. Pac., 115, 763

Churazov E., Brüggen M., Kaiser C.R., Böhringer H., Forman W., 2001, ApJ, 554, 261

Cora S.A., Tornatore L., Tozzi P., Dolag K., 2008, MNRAS, 386, 96

De Grandi S., Molendi S., 2001, ApJ, 551, 153

De Grandi S., Ettori S., Longhetti M., Molendi S., 2004, A\&A, 419,7

de Plaa J., 2013, Astronomische Nachrichten, 334, 416

de Plaa J., Werner N., Bleeker J.A.M., Vink J., Kaastra J.S., Méndez M., 2007, A\&A, 465, 345

Dolag K., Borgani S., Murante G., Springel V., 2009, MNRAS, 399, 497

Dolag K., Mevius E., Remus R.S., 2017, Galaxies, 5, 35

Ettori S., Gastaldello F., Gitti M., O’Sullivan E., Gaspari M., Brighenti F., David L., Edge A.C., 2013, A\&A, 555, A93

Ettori S., Baldi A., Balestra I., Gastaldello F., Molendi S., Tozzi P., 2015, A\&A, 578, A46

Ezer C., Bulbul E., Nihal Ercan E., Smith R.K., Bautz M.W., Loewenstein M., McDonald M., Miller E.D., 2017, ApJ, 836, 110

Fabjan D., Tornatore L., Borgani S., Saro A., Dolag K., 2008, MNRAS, 386, 1265

Fabjan D., Borgani S., Tornatore L., Saro A., Murante G., Dolag K., 2010, MNRAS, 401, 1670

Finoguenov A., David L.P., Ponman T.J., 2000, ApJ, 544, 188

Fujita Y., Tawa N., Hayashida K., Takizawa M., Matsumoto H., Okabe N., Reiprich T.H., 2008, Pub. Astron. Soc. Japan, 60, S343

Gaspari M., Sądowski A., 2017, ApJ, 837, 149
Gaspari M., Melioli C., Brighenti F., D’Ercole A., 2011, MNRAS, 411,349

Gaspari M., Brighenti F., Temi P., 2012, MNRAS, 424, 190

Haardt F., Madau P., 2001, in D.M. Neumann, J.T.V. Tran, eds., Clusters of Galaxies and the High Redshift Universe Observed in $X$-rays

Hunt L., Dayal P., Magrini L., Ferrara A., 2016, MNRAS, 463, 2020

Karakas A.I., 2010, MNRAS, 403, 1413

Komatsu E., et al., 2011, ApJS, 192, 18

Kravtsov A., Vikhlinin A., Meshscheryakov A., 2014, ArXiv eprints

Kravtsov A.V., Borgani S., 2012, ARA\&A, 50, 353

Leccardi A., Molendi S., 2008, A\&A, 487, 461

Leccardi A., Rossetti M., Molendi S., 2010, A\&A, 510, A82

Mantz A.B., Allen S.W., Morris R.G., Simionescu A., Urban O., Werner N., Zhuravleva I., 2017, ArXiv e-prints

Martizzi D., Hahn O., Wu H.Y., Evrard A.E., Teyssier R., Wechsler R.H., 2016, MNRAS, 459, 4408

Matsushita K., Sato T., Sakuma E., Sato K., 2013, Pub. Astron. Soc. Japan, 65

Maughan B.J., Jones C., Forman W., Van Speybroeck L., 2008, ApJS, 174, 117-135

McCarthy I.G., et al., 2010, MNRAS, 406, 822

McDonald M., et al., 2016, ApJ, 826, 124

Mernier F., de Plaa J., Pinto C., Kaastra J.S., Kosec P., Zhang Y.Y., Mao J., Werner N., 2016a, A\&A, 592, A157

Mernier F., et al., 2016b, ArXiv e-prints

Mernier F., et al., 2017, A\&A, 603, A80

Molendi S., Eckert D., De Grandi S., Ettori S., Gastaldello F., Ghizzardi S., Pratt G.W., Rossetti M., 2016, A\&A, 586, A32

Mushotzky R., Loewenstein M., Arnaud K.A., Tamura T., Fukazawa Y., Matsushita K., Kikuchi K., Hatsukade I., 1996, ApJ, 466, 686

Nandra K., et al., 2013, ArXiv e-prints

Nomoto K., Kobayashi C., Tominaga N., 2013, ARA\&A, 51, 457

Overzier R.A., 2016, A\&A Rev., 24, 14

Padovani P., Matteucci F., 1993, ApJ, 416, 26

Planelles S., Borgani S., Fabjan D., Killedar M., Murante G., Granato G.L., Ragone-Figueroa C., Dolag K., 2014, MNRAS, 438, 195

Planelles S., Schleicher D.R.G., Bykov A.M., 2016, Large-Scale Structure Formation: From the First Non-linear Objects to Massive Galaxy Clusters, pp. 93-139

Planelles S., et al., 2017, MNRAS, 467, 3827

Rasia E., Mazzotta P., Bourdin H., Borgani S., Tornatore L., Ettori S., Dolag K., Moscardini L., 2008, ApJ, 674, 728-741

Rasia E., et al., 2015, ApJ, 813, L17

Rasmussen J., Ponman T.J., 2009, MNRAS, 399, 239

Rebusco P., Churazov E., Böhringer H., Forman W., 2005, MNRAS, 359, 1041

Rebusco P., Churazov E., Böhringer H., Forman W., 2006, MNRAS, 372, 1840

Renzini A., Andreon S., 2014, MNRAS, 444, 3581

Romano D., Karakas A.I., Tosi M., Matteucci F., 2010, A\&A, 522, A32

Sakuma E., Ota N., Sato K., Sato T., Matsushita K., 2011, Pub. Astron. Soc. Japan, 63, S979

Sánchez S.F., et al., 2013, A\&A, 554, A58

Sato K., Matsushita K., Ishisaki Y., Yamasaki N.Y., Ishida M., Sasaki S., Ohashi T., 2008, Pub. Astron. Soc. Japan, 60, S333 
Simionescu A., Werner N., Finoguenov A., Böhringer H., Brüggen M., 2008, A\&A, 482, 97

Simionescu A., Werner N., Böhringer H., Kaastra J.S., Finoguenov A., Brüggen M., Nulsen P.E.J., 2009, A\&A, 493, 409

Simionescu A., Werner N., Urban O., Allen S.W., Ichinohe Y.,

Zhuravleva I., 2015, ApJ, 811, L25

Springel V., 2005, MNRAS, 364, 1105

Springel V., Hernquist L., 2003, MNRAS, 339, 289

Steinborn L.K., Dolag K., Hirschmann M., Prieto M.A., Remus R.S., 2015, MNRAS, 448, 1504

Thielemann F.K., et al., 2003, in W. Hillebrandt, B. Leibundgut, eds., From Twilight to Highlight: The Physics of Supernovae, p. 331

Thölken S., Lovisari L., Reiprich T.H., Hasenbusch J., 2016, A\&A, 592, A37

Tornatore L., Borgani S., Matteucci F., Recchi S., Tozzi P., 2004, MNRAS, 349, L19

Tornatore L., Borgani S., Dolag K., Matteucci F., 2007, MNRAS, 382,1050

Tremonti C.A., et al., 2004, ApJ, 613, 898

Truong N., et al., 2018, MNRAS, 474, 4089

Urban O., Werner N., Allen S.W., Simionescu A., Mantz A., 2017, ArXiv e-prints

Villaescusa-Navarro F., et al., 2016, MNRAS, 456, 3553

Vogelsberger M., et al., 2017, ArXiv e-prints

Werner N., Urban O., Simionescu A., Allen S.W., 2013, Nature, 502,656

Wiersma R.P.C., Schaye J., Smith B.D., 2009, MNRAS, 393, 99

Woosley S.E., Weaver T.A., 1995, ApJS, 101, 181

Yates R.M., Thomas P.A., Henriques B.M.B., 2017, MNRAS, 464, 3169

\section{APPENDIX A: BEHAVIOUR OF DIFFERENT CLUSTERS}

We performed a similar analysis to the one shown for D2 in the previous sections on the representative subsample of clusters, for which the tracking of the metal origin is available, listed in Table 1 Far from having any statistical purpose, the aim of this test is purely to explore whether dependencies of our results on the system mass or central thermal properties exist or not.

Observational findings, as well as numerical investigations (e.g. Yates et al. 2017; Barnes et al. 2017, Vogelsberger et al. 2017. Dolag et al. 2017), have shown that the chemical properties of the ICM at large cluster-centric distances is extremely uniform across different clusters, and is essentially independent on the specific thermal properties of the cluster core, such as cool-coreness, which cause instead a larger variation in the core, from cluster to cluster. Here we further explore whether this homogeneity regards as well the origins of the present-day outskirts metal content.

With the same criteria used for D2, we select and track back in time the gas in the outskirts of the present-day clusters in Table 1 (namely the hot-phase ICM enclosed in the spherical shell between $0.75 R_{200}$ and $R_{200}$ ) and we find no remarkable differences between D2 and the other three systems. The distribution of the iron abundance of the selected gas at $z=0$ and its evolution at higher redshifts presents in fact the same features than those presented in Figs. 2 and 10 More specifically, the characteristic peak at high iron abundances is found in all the four clusters (either small or massive, (CC or NCC) and is already clearly present at $z=2$,

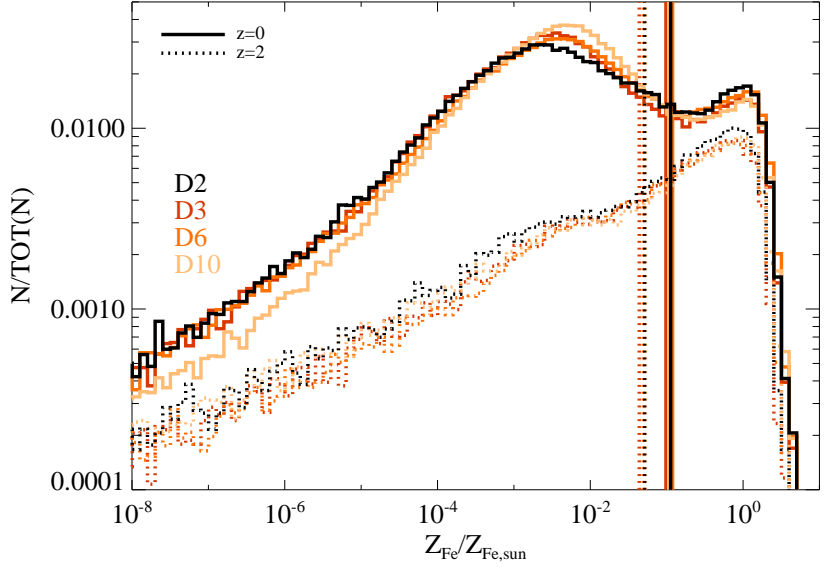

Figure A1. Distribution of the Fe abundance (w.r.t. solar values by ANGR89) for the hot-phase gas particles selected to reside within [0.751] $R_{200}$ at $z=0$ and tracked back in time, shown for $z=0$ (solid lines) and $z=2$ (dotted lines) for all the four clusters as in the legend. Vertical lines correspond to the MW value of each distribution.

indicating that that gas was already significantly enriched by then. This is visible from Fig. A1

Furthermore, the MW iron abundance of the tracked gas always moderatly varies between 0.1 and 0.2 , if enclosed within some halo in the region, whereas it increases from $\sim 0.02-0.03$ at $z=2$ to $\sim 0.1-0.15$ at $z=0$ for the gas in the diffuse component. When the gas traced is selected on the base of its present-day iron abundance, we also find similar results to those discussed for D2. Also, the contribution to the metal mass budget of the selected gas is always dominated by SNII, with similar proportions than in D2. The iron-poor gas, instead, is also in all these cases primarily polluted by SNIa at all redshifts, indicating a late and limited enrichment far from the active star-formation regions.

This further confirms the uniformity of the metal enrichment in the outskirts of clusters at $z=0$, independently of their mass and central thermal properties. Also, they support the idea of an early enrichment to which both SNIa and SNII have contributed, mainly within small high-redshift haloes from which the metal-rich gas has been pushed out by early AGN feedback, able to overcome their still shallow potential wells. The pre-enriched gas was then accreted from both the diffuse component as well as with the merging of the surrounding haloes during the formation of the cluster. Essentially, the backward tracking analysis adds an important piece to the puzzle: not only the present-day metal distribution of cluster outskirts, but also the origin of the metals and their evolution in the ICM at the periphery of clusters of different mass and thermal core properties share remarkably similar features.

We note that minor differences among the four clusters analysed, mainly in the values of MW $Z_{\mathrm{Fe}}$ of the tracked gas residing within surrounding haloes, rather correspond to differences in their dynamical history and mass assembly. For instance this can be related to the presence of - and merging with - systems, in the proto-cluster environment, that have comparable mass to the main progenitor, rather than the accretion of smaller-mass (by a factor of 2 or more) haloes such as in the case of D2. 